\title{
rf deflector design of the CLIC test facility CTF3 delay loop and beam loading effect analysis
}

\author{
David Alesini and Fabio Marcellini \\ INFN Laboratori Nazionali di Frascati, P.O. Box 13, I-00044, Frascati (Roma), Italy
}

(Received 23 September 2008; published 10 March 2009)

\begin{abstract}
Beam injection and extraction in the delay loop of the CLIC Test Facility 3 (CTF3) for recombination of adjacent bunch trains, is performed by a specially designed $\mathrm{rf}$ deflector. A standing wave structure has been chosen for this purpose. Three possible solutions have been investigated, and a comparative analysis is presented. All of them satisfy the essential requirements of the system up to the maximum foreseen energy with the existing klystron. The final design of the rf deflector consists of two identical cavities connected to the rf power source through a hybrid junction that equally splits the power and isolates the klystron from reflections. The rf deflector design, the results of electromagnetic simulations, and the low level rf measurements are illustrated. The impact of beam loading in the rf deflectors on the transverse beam dynamics is also analyzed. The general expression of the single passage transverse wakefield is obtained and a dedicated tracking code has been written to study the multibunch multiturn effects. A complete analysis for different machine parameters and injection errors is presented and discussed. These numerical simulations indicate a tolerable beam emittance growth due to the transverse wakefield in the rf deflectors.
\end{abstract}

DOI: 10.1103/PhysRevSTAB.12.031301

PACS numbers: 29.20.db, 29.27.Bd, 42.79.Fm, 42.60.Da

\section{INTRODUCTION}

CTF3 [1] is the third test facility of the CLIC (Compact Linear Collider) project $[2,3]$. The CTF3 main goals were to demonstrate the feasibility of the generation of the $30 \mathrm{GHz}$ rf power through the bunch recombination technique as well as the acceleration of a probe beam with $30 \mathrm{GHz}$ rf cavities at very high gradient $(\sim 150 \mathrm{MV} / \mathrm{m})$. Recently, the target frequency generation has been changed from 30 to $12 \mathrm{GHz}$ [4].

The conceptual layout of CTF3 is shown in Fig. 1. The frequency multiplication by the novel bunch interleaving technique is one of the most important issues to be tested. In the facility a long $(\approx 1.4 \mu \mathrm{s})$ train of $20 \mathrm{~cm}$ spaced short bunches (drive beam) is converted into a short (140 ns) train of $2 \mathrm{~cm}$ spaced bunches. In the first stage the pulse is compressed by a factor of 2 by means of a transverse rf deflector (RFD) at $1.499 \mathrm{GHz}$ and a $42 \mathrm{~m}$ circumference delay loop (DL). An $84 \mathrm{~m}$ circumference combiner ring (CR) is used in the second stage to further compress the pulse by a factor $5 .^{1}$ The process of beam compression in the DL is illustrated in Fig. 2. The beam coming from the LINAC is an alternate sequence of socalled "even" and "odd" trains, which have a phase difference of $180^{\circ}$ with respect to the $1.499 \mathrm{GHz}$ of the RFD. This sequence of $140 \mathrm{~ns}$ long trains is realized by a prebunching system. The rf deflector kicks with the same amplitude but opposite sign the incoming "even" and "odd" trains. The "even" trains are injected into the DL and, by properly setting the DL circumference, after one

\footnotetext{
${ }^{1}$ Because of the new target frequency of $12 \mathrm{GHz}$ the compression factor in the $\mathrm{CR}$ is now four instead of five.
}

turn they are extracted and interleaved with the following "odd" trains. The deflection given by the RFD to the bunches is $\pm 15 \mathrm{mrad}$ while the beam energy is up to $300 \mathrm{MeV}$. The main beam parameters at the injection/ extraction of the DL are reported in Table I.

Both the RFD design and the study of its interaction with the beam have been crucial points in the DL project.

For the RFD structure some possible solutions have been considered (Sec. II A). The chosen one consists of two identical standing wave (SW) cavities connected to the rf power source through a hybrid junction that equally splits the power and isolates the klystron from reflections (Secs. II B, II C, and II D). Such design, typically used in the SLED cavities [6], avoids the use of the circulator and makes the structure absolutely innovative and nonconventional. Good results have been obtained from low power measurements (Sec. IIE) and high power tests [5], which have confirmed the predictions of the electromagnetic simulations and the feasibility of such new scheme.

The study of the RFD interaction with the beam in terms of beam loading has been also important to demonstrate the compatibility of the RFD design with the beam dynamics requirements. The importance of such analysis has also been pointed out in [7] where the problem of beam loading in the traveling wave RFD of the CTF3 combiner ring has been carefully investigated. Transverse emittance growth or particle loss could, as a matter of fact, reduce the effectiveness of power conversion at $30 \mathrm{GHz}$. The analysis starts from obtaining the expression of the transverse single passage wakefield in a SW deflecting cavity (Sec. III A) for a parabolic trajectory of a leading particle in the RFD. The obtained formulas are not given in literature and can be useful to evaluate the beam loading effects in a general 


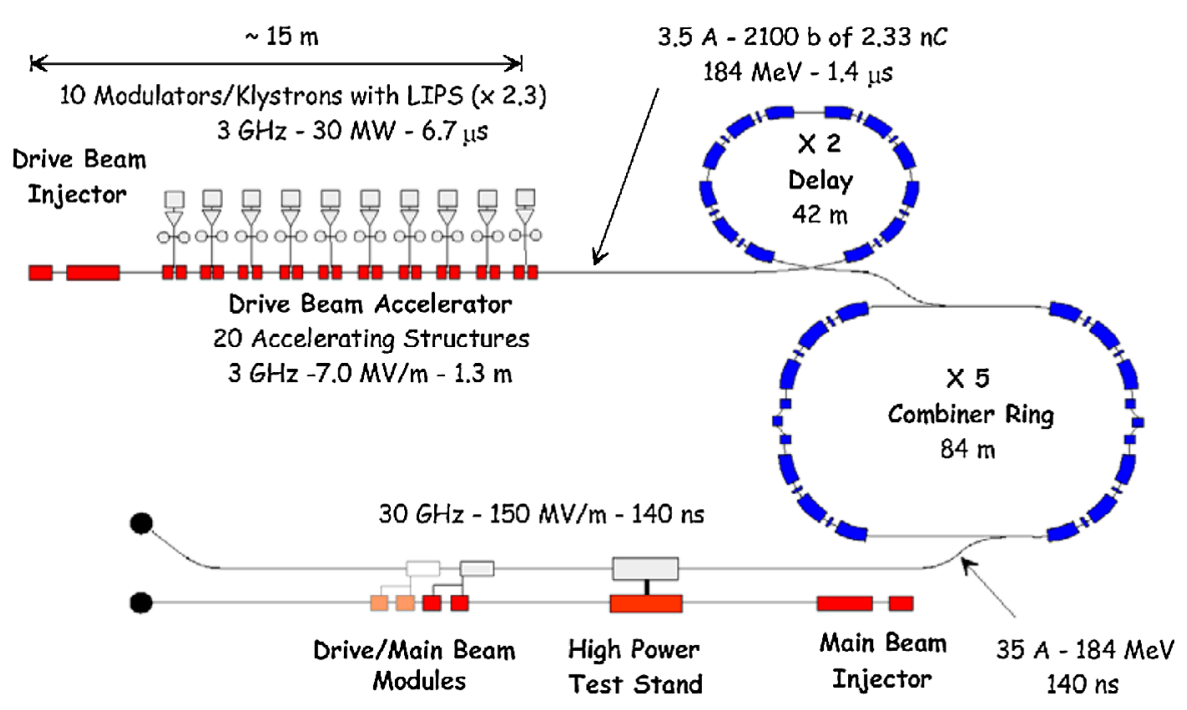

FIG. 1. (Color) Conceptual layout of CTF3.

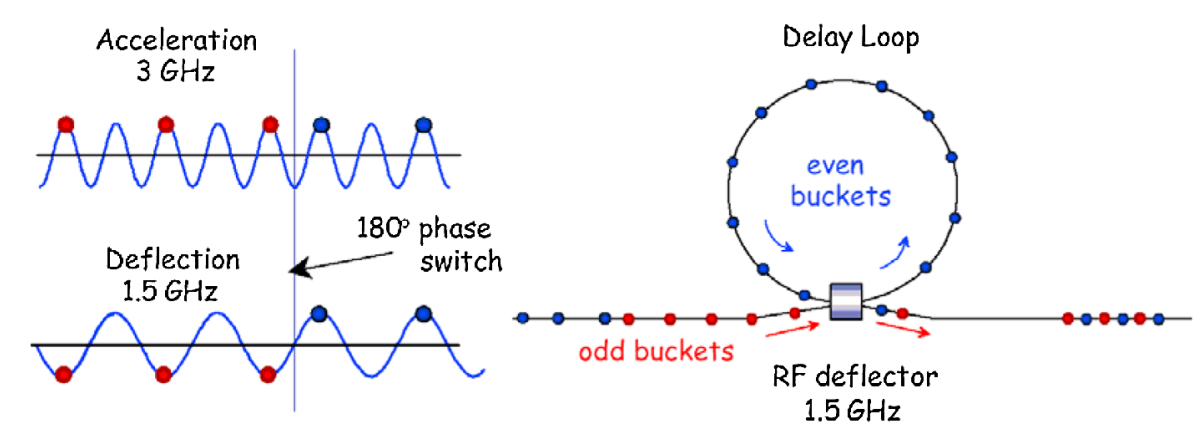

FIG. 2. (Color) Sketch of the bunch frequency multiplication in the DL.

TABLE I. Main beam parameters at the injection/extraction of the $\mathrm{DL}^{\mathrm{a}}$.

\begin{tabular}{lcc}
\hline \hline Energy $(E)$ & & $150[\mathrm{MeV}]$ \\
Bunch charge $\left(Q_{b}\right)$ & $2.33[\mathrm{nC}]$ \\
Bunch length $\left(\sigma_{z}\right)$ & $1-3[\mathrm{~mm}]$ \\
Beam emittance ${ }^{\mathrm{b}}(\varepsilon)$ & $\sim 0.4[\mathrm{~mm} \mathrm{mrad}]$ \\
Number of bunches per pulse $\left(N_{b}\right)$ & 2100 \\
Total pulse duration & & $1.36[\mu \mathrm{s}]$ \\
Before recombination & Number of trains per pulse $\left(N_{\text {pinj }}\right)$ & 10 \\
& Bunch separation $\left(\tau_{B}\right)$ & $0.66[\mathrm{~ns}]$ \\
After recombination & Number of trains per pulse $\left(N_{\text {pextr }}\right)$ & 5 \\
& Bunch separation $\left(\tau_{A}\right)$ & $0.33[\mathrm{~ns}]$ \\
\hline \hline
\end{tabular}

${ }^{\mathrm{a}}$ All parameters are those reported in [1]. Concerning the energy, we considered the lower energy case, corresponding to the present energy of operations [5], even if there are options up to $300 \mathrm{MeV}$.

${ }^{\mathrm{b}}$ The emittance is a transverse projected rms emittance not normalized, defined by

$$
\varepsilon=\sqrt{\left\langle x^{2}\right\rangle\left\langle x^{\prime 2}\right\rangle-\left\langle x x^{\prime}\right\rangle^{2}}
$$

where $x, x^{\prime}$ are the position and angle of particles in the transverse phase space and \langle\rangle is the average value over the bunch. 
TABLE II. Deflecting cavity design parameters.

\begin{tabular}{lc}
\hline \hline Maximum input power & $20[\mathrm{MW}]$ \\
Frequency of operation $\left(f_{\mathrm{RF}}\right)$ & $1.4995[\mathrm{GHz}]$ \\
Loaded quality factor $\left(Q_{L}\right)$ & $\sim 3300$ \\
Loaded transverse shunt impedance $\left(R_{T}\right)^{\mathrm{a}}$ & $\sim 0.5[\mathrm{M} \Omega]$ \\
Cavity maximum deflecting voltage $\left(V_{T}\right)$ & $4.5[\mathrm{MV}]$ \\
Cavity filling time $\left(\tau_{F}\right)$ & $\sim 0.7[\mu \mathrm{s}]$ \\
\hline \hline
\end{tabular}

${ }^{\mathrm{a}}$ We have defined the transverse shunt impedance by the formula

$$
R_{T}=\frac{\left|\int_{0}^{L_{c}}\left(c B_{x}+E_{y}\right) e^{j \omega_{\mathrm{rf}} z / c} d z\right|^{2}}{2 P_{\mathrm{tot}}},
$$

where $B_{x}$ and $E_{y}$ are the magnetic and electric transverse field components (with the proper phase), $z$ is the beam traveling direction, $L_{c}$ is the cavity length, $P_{\text {tot }}$ is the total dissipated power in the cavity and external load, and $\omega_{\mathrm{rf}}=2 \pi f_{\mathrm{rf}}$ the angular frequency.

deflecting SW cavity. Then, a dedicated tracking code has been written to study the multibunch multiturn effects on the transverse beam dynamics (Sec. III B) both on the horizontal and vertical planes (Secs. III C and III D) of DL.

The final operational performances and the conclusions are given in Secs. IV and V, respectively.

\section{RFD DESIGN}

\section{A. Design options}

The available power source to feed the RFD is a klystron delivering $20 \mathrm{MW} 5 \mu \mathrm{s}$ long pulses. According to the beam deflection specification, a traveling wave (TW) deflector working on the $\mathrm{TM}_{11}$-like mode should be a structure longer than $1 \mathrm{~m} \mathrm{[8]}$, but this is not compatible with the available space and deflected beam trajectories. For these reasons the adoption of a more compact and efficient SW cavity operating on the $\mathrm{TM}_{110}$ mode was preferred $[9,10]$.

The major drawback of this choice is that the voltage filling time of a resonant cavity is generally slow with respect to the rf pulse length $(5 \mu \mathrm{s})$ and, therefore, the deflecting field is not constant during the passage of the bunch train in the cavity and different bunches get different kicks. The upper limit of deflection angle spread between the head and the tail of the train is $0.5 \%$. This requirement sets a corresponding upper limit for the cavity filling time and then the loaded quality factor $\left(Q_{L}\right)$. The maximum $Q_{L}$ is $\sim 3300$ and it has been assumed as cavity design parameter. $^{2}$ We report the final cavity design parameters in Table II.

Considering a simple pill-box shaped cavity, the required transverse impedance can be achieved with a two cell SW structure. Three possible solutions for the SW

\footnotetext{
${ }^{2}$ It is not convenient to further decrease the value of $Q_{L}$ unless to decrease the shunt impedance, i.e., the efficiency of the deflector.
}

(a)

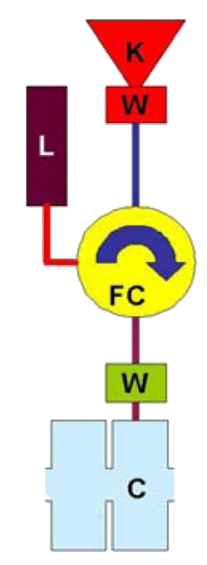

(b)

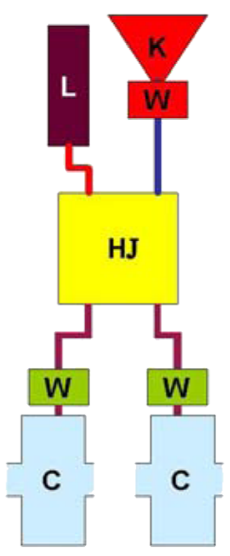

(c)

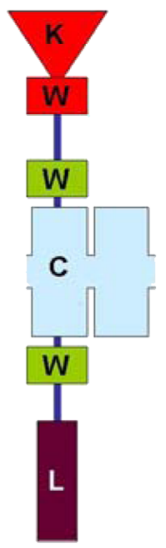

FIG. 3. (Color) Different design options for the RFD ( $\mathrm{K}$ is the klystron; FC is the ferrite circulator; $\mathrm{L}$ is the load; HJ is the hybrid junction; $\mathrm{C}$ is the $\mathrm{SW}$ cavity; $\mathrm{W}$ is the vacuum tight ceramic window).

deflector have been taken into account [11] and their layouts are schematically shown in Fig. 3.

(a) The first solution [Fig. 3(a)] is the most standard one. The two cavity structure is excited by a single coupler and the coupling coefficient itself is designed to reach the desired $Q_{L}$. Typical values of the unloaded quality factor $\left(Q_{0}\right)$ of deflecting cavities at the working frequency of $1.5 \mathrm{GHz}$ are $\sim 22000$ and, therefore, a coupling coefficient $(\beta)$ of $\sim 5.6$ is required to reach the desired $Q_{L}$. This implies that the SW cavity reflects power back to the source not only during the initial and final pulse transients but even when the cavity has reached a stationary regime. As an example we report in Fig. 4 the time dependence of the rf reflected power for an input pulse with $1 \mu \mathrm{s}$ rise

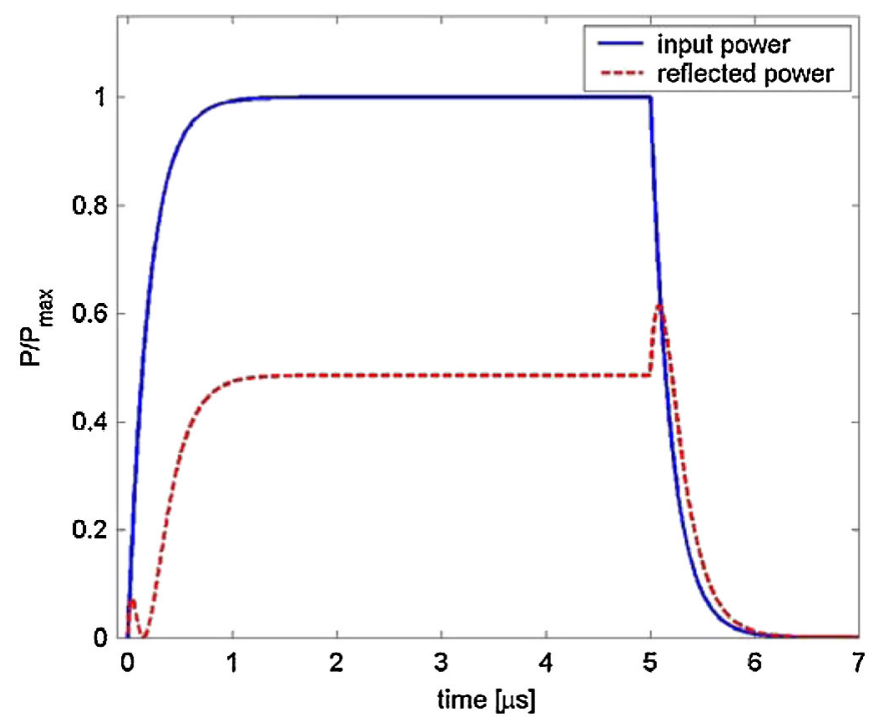

FIG. 4. (Color) Time domain cavity response to a step input in case of $\beta=5.6$ (blue: rf input pulse; red: cavity reflected power). 
time. The reflected power in the stationary regime is $\sim 50 \%$ of the incident power but during the transients it can be more than $100 \%$ depending on the rise time. In this case, therefore, a circulator must be foreseen to protect the klystron from reflections.

(b) The second solution [Fig. 3(b)] is based on the SLED principle used in the LINAC technology [6] and consists of two cavities coupled through a hybrid junction (HJ) [12]. The power reflected by the cavities adds out of phase at the klystron port while adds in phase at the port connected to the load. As a consequence, no reflected power results at the klystron port. Finally, although two ceramic windows instead of one are necessary in this scheme, they can be dimensioned to be able to support half of the rf power with respect to option (a).

(c) The third solution [Fig. 3(c)] is a double cell cavity with two different coupling ports: one is connected to the klystron and the other to the load. In this case the two coupling coefficients can be set to have no reflection at the klystron port at the working frequency. This means that the klystron "sees" a cavity with $\beta=1$. Even if the reflection coefficient at the working frequency is equal to zero, when the cavity is used in a pulsed regime the level of reflected rf power shows peaks of reflections during the transients. The height of these peaks depends on the pulse rise and decay times. As an example, Fig. 5 shows the time dependence of the $\mathrm{rf}$ reflected power for a typical input pulse with rise and decay times of $1 \mu \mathrm{s}$. Even if in this case the amount of reflected power is significantly lower than in case (a), the peaks of reflections are just below the tolerable klystron threshold and the klystron itself is subject to repeated reflected pulses which can become dangerous in a long term operation.

From all previous considerations and taking into account that high power circulators are expensive devices com-

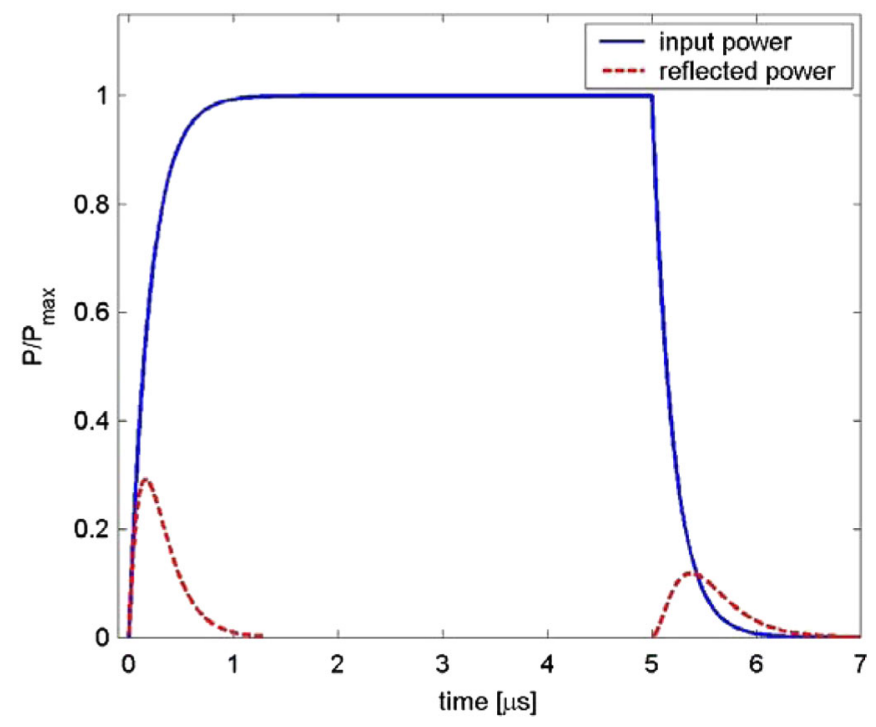

FIG. 5. (Color) Time domain cavity response to a step input in case of $\beta=1$ (blue: rf input pulse; red: cavity reflected power).

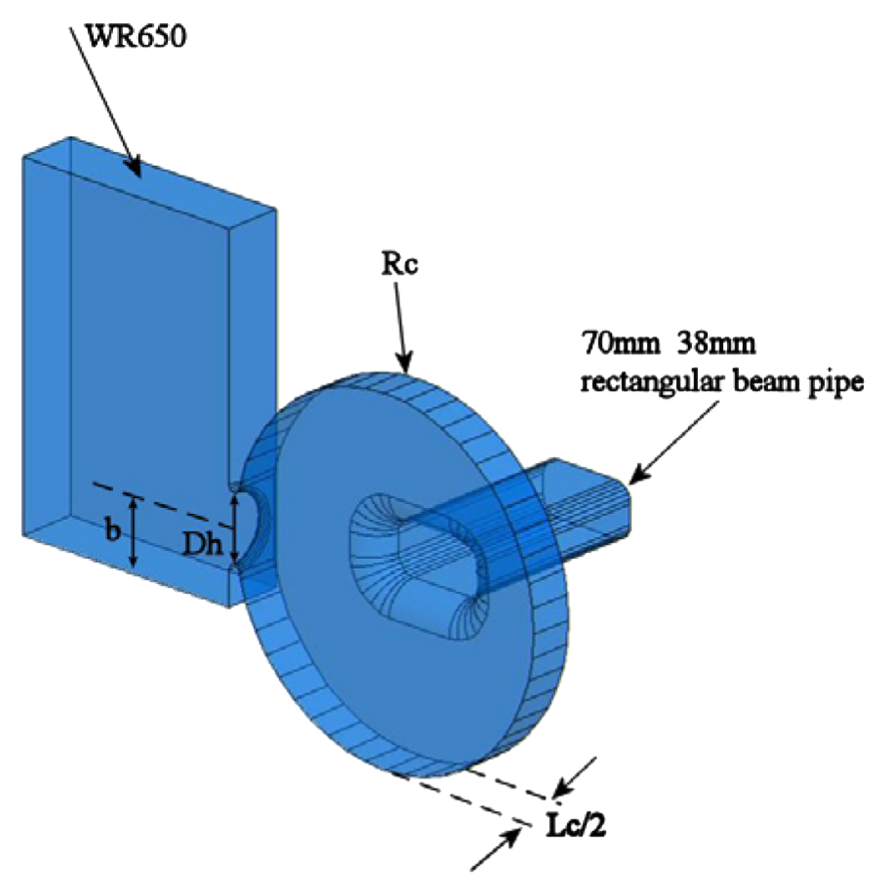

FIG. 6. (Color) Single cell cavity geometry.

pared to HJs, option (b) has been adopted as final deflecting structure scheme for the CTF3 DL.

\section{B. Single cavity design}

The single cell cavity profile is shown in Fig. 6. The cavity has been designed starting from a simple pill-box shape. It is coupled to a rectangular waveguide (WR650, the same standard of the klystron output) through a hole in which the diameter and thickness have been chosen to obtain the desired $Q_{L}$. The cavity frequency tuning is realized by a cylinder penetrating in the cell while the field in the cavity is probed by two small antennas. The whole electromagnetic design has been done using HFSS [13]. Figure 7 shows the electric and magnetic field profiles on axis. The main dimensions and achieved parameters of the cavity are reported in Table III.

Parasitic modes of the cavity can be excited by the beam. By simulations we have verified that the resonant frequencies of the most dangerous modes (monopoles and dipoles) are far enough from the beam power spectrum lines and in a single beam passage the effects on longitudinal and transverse beam dynamics are negligible. In particular, the $\mathrm{TM}_{110}$ mode with vertical polarization is shifted away by more than $40 \mathrm{MHz}$ from the horizontal one because of the asymmetry introduced by the coupling hole and rectangular beam pipe.

\section{Complete deflecting system}

The HJ has been also designed by HFSS and the whole deflecting system with the hybrid and two cavities has been finally simulated [11] and is shown in Fig. 8. 

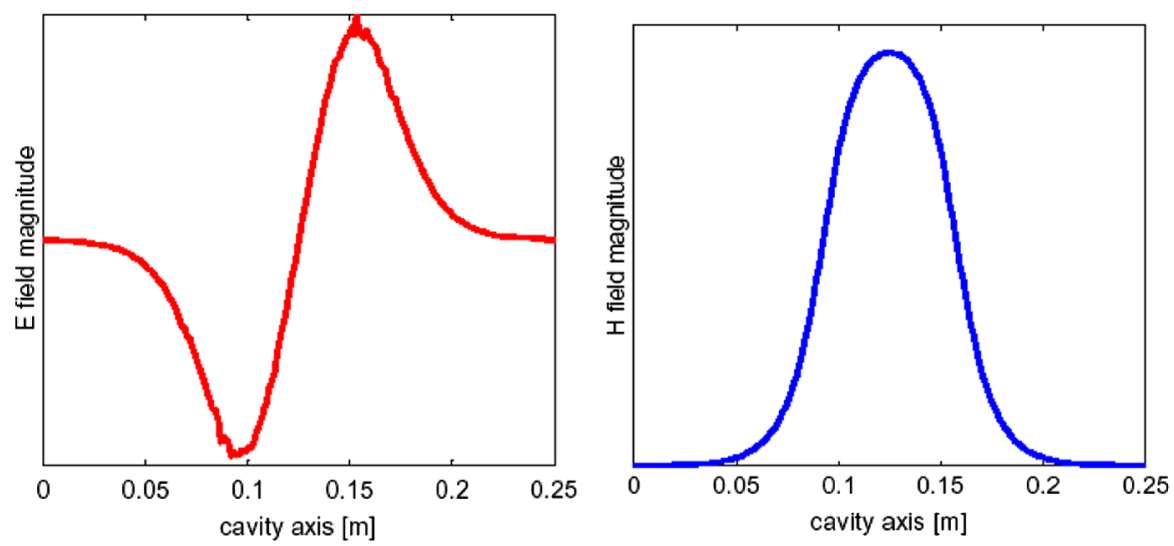

FIG. 7. (Color) Electric and magnetic field profiles on cavity axis (HFSS result).

TABLE III. Single cell deflecting cavity main dimensions and achieved parameters.

\begin{tabular}{lc}
\hline \hline Cavity length $(L c)$ & $60[\mathrm{~mm}]$ \\
Cavity radius $(R c)$ & $120[\mathrm{~mm}]$ \\
Hole diameter $(D h)$ & $10[\mathrm{~mm}]$ \\
Distance $(b)$ & $30[\mathrm{~mm}]$ \\
Cylinder tuning diameter & $5[\mathrm{~mm}]$ \\
Tuning sensitivity & $d f_{\mathrm{rf}} / d p=150 \mathrm{kHz} / \mathrm{mm}$ \\
Unloaded quality factor $\left(Q_{0}\right)$ & 22000 \\
Coupling coefficient $(\beta)$ & 5.6 \\
Transverse shunt impedance $\left(R_{T}\right)$ & $0.625[\mathrm{M} \Omega]$ \\
Average dissipated power into the & $2.5 \mathrm{~kW}$ \\
$\quad$ cavity @ $10 \mathrm{MW}$ of input power & $\sim 0.7[\mu \mathrm{s}]$ \\
Cavity filling time $\left(\tau_{F}\right)$ & \\
\hline \hline
\end{tabular}

Since the hybrid splits the power coming from the klystron at the two ports connected to the cavities with a phase difference of $90^{\circ}$, the two cavities have to be placed at a distance $(L)$ equal to an odd multiple integer of the radio frequency wavelength divided by four $\left(\lambda_{\mathrm{rf}} / 4\right)$ in order to have the two deflections in phase. From the point of view of available space and beam trajectories inside the deflectors, this distance should be as small as possible

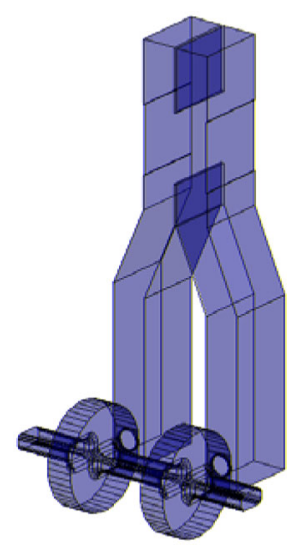

FIG. 8. (Color) HFSS model of the whole deflector structure. while, from the point of view of possible excitation of coupled modes, this distance should be as large as possible. The best compromise has been found to be $L=5 \lambda_{\mathrm{rf}} / 4=$ $250 \mathrm{~mm}$.

The whole deflector structure, including the two cavities and the hybrid junction, was finally simulated and an acceptable value (below $-20 \mathrm{~dB}$ ) of reflection coefficient at the klystron port was obtained.

\section{Cavities fabrication and final assembling}

All the waveguide components of the system have been manufactured by Mega Industries [14] and are WR650 standard products. They are reinforced to stand the 3 bar SF6 overpressure. In particular, the HJ has been designed to fit the distance between the two cavities in the final layout. Four directional couplers have been inserted close to the cavities, the load, and the klystron to serve as a monitor of the forward and reflected powers.

Figure 9 shows the two cavities and the final assembly of the deflecting system. The two vacuum windows separating the SF6 from the vacuum environment are also visible. They have been manufactured by Thales [15] on the same design of the window on the klystron output. In the two branches between the cavity couplers and the HJ, the rf wave configuration is partially standing, being the cavities overcoupled. Then, for safety reasons, the window position has been chosen in correspondence of a minimum of the fields along the waveguide line.

The two cavities have been fabricated in Poland by the A. Soltan Institute [16] and are made of OFHC copper. They are provided with five water pipes for the temperature stabilization $\left(30 \pm 0.1^{\circ} \mathrm{C}\right)$ water.

\section{E. rf measurements and final tuning}

Each single component of the whole system has been characterized by rf measurements [17] to verify its correspondence with the design specification and with the results obtained from the HFSS simulation code. Moreover, to optimize the isolation of the klystron from the reflected 


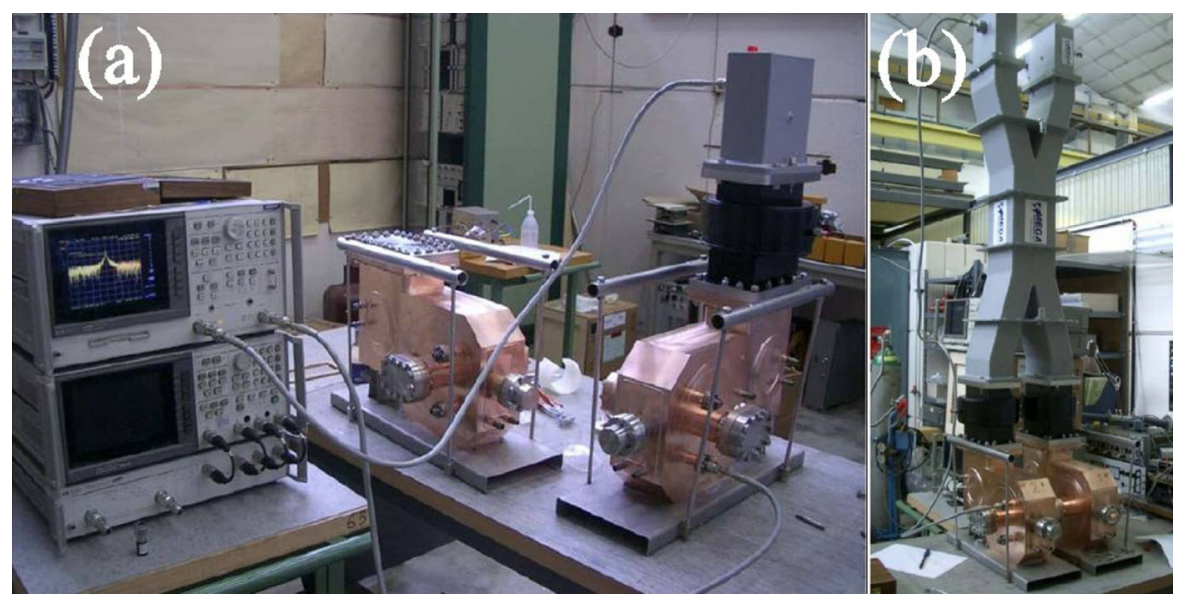

FIG. 9. (Color) (a) Two rf deflecting cavities on the test bench, the cavities are made of OFHC copper. (b) Assembled cavities with HJ, ceramic window, and waveguides.

power, all components connected to the two lower branches of the HJ (cavities, windows, bends, and drift waveguides) must be as identical as possible, so that the impedance seen from those ports of the HJ is identical. For the same reason, an accurate tuning of the resonant frequency of the cavity operating mode was necessary.

As a first step, the frequencies of the two cavities have been separately measured and tuned. The final tuning has been done with all components assembled in vacuum condition and at nominal temperature. The $Q_{L}$ values of the cavities have been also measured giving $Q_{L 1}=3100$ and $Q_{L 2}=3400$ in agreement with the mechanical tolerance specification in the fabrication process. Figure 10 reports the reflection coefficient at the $\mathrm{HJ}$ port connected to the klystron showing an excellent isolation of the klystron from cavity reflections. Also the amplitude and phase signal from the klystron port to the cavities has been measured showing that the phase balance is within $0.25^{\circ}$ and the amplitude differs by a few percent.

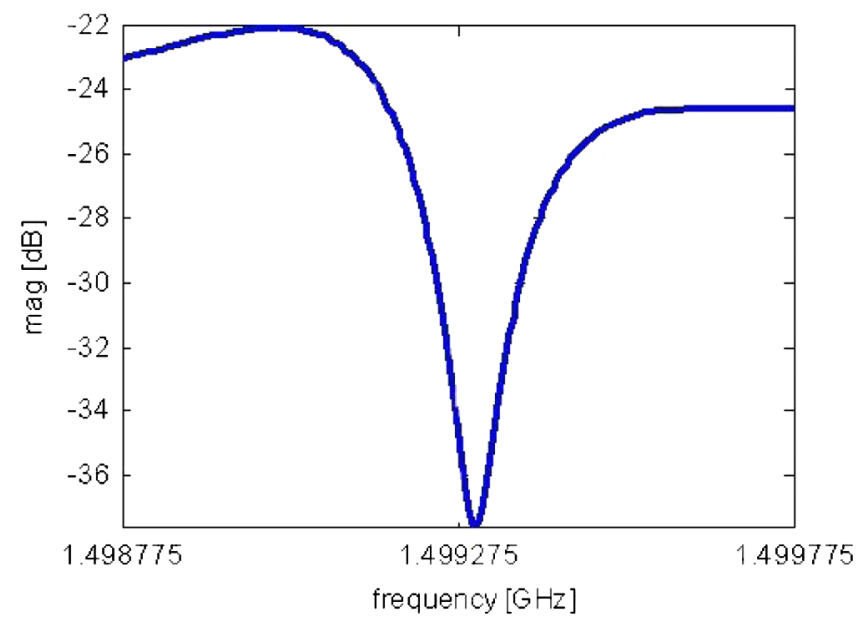

FIG. 10. (Color) $S_{11}$ at the HJ input port connected to the klystron after tuning.

\section{EFFECTS OF THE BEAM LOADING IN THE RF DEFLECTOR}

\section{A. Single passage wakefield}

Let us consider the case of a simple pill-box cavity operating on the $\mathrm{TM}_{110}$ fundamental deflecting mode [Fig. 11(a)]. The field components in the frequency domain, calculated on the deflection plane $(x, z)$, are given by [18]

$\underline{E}_{D}=\left\{\begin{array}{l}E_{D z}=E_{0} J_{1}\left(p_{11} x / a\right) \\ E_{D r}=E_{D x}=0 \\ E_{D \vartheta}=E_{D y}=0\end{array}\right.$
$\underline{B}_{D}=\left\{\begin{array}{l}B_{D z}=0 \\ B_{D r}=B_{D x}=0 \\ B_{D \vartheta}=B_{D y}=-j \omega_{\mathrm{rf}} a / p_{11} c^{2} E_{0} J_{1}^{\prime}\left(p_{11} x / a\right),\end{array}\right.$

where $p_{11}$ is the first zero of the $J_{1}$ Bessel function and $\omega_{\text {rf }}$ the angular rf frequency. In (1) we have neglected all field components given by the presence of the beam pipe tubes.
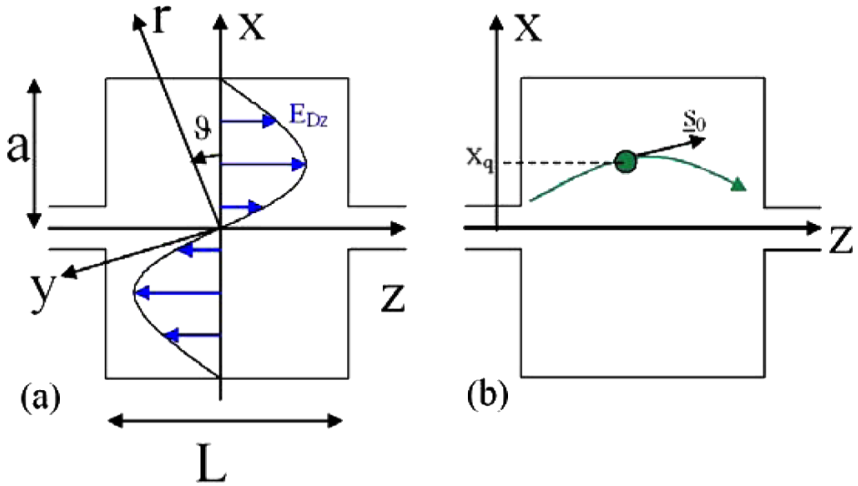

(b)

FIG. 11. (Color) (a) Sketch of a RFD cavity with the $E_{D z}$ field (blue vectors). (b) Sketch of a particle passing through the RFD on the deflection plane $(x, z)$. 
The sketch of a charge $q$ moving inside the rf deflector on the plane $(x, z)$, is given in Fig. 11(b). In the figure $\underline{s}_{0}$ is the unit vector tangent to the particle trajectory and $x_{q}$ is the transverse position of the particle.

Appendix A shows in detail the calculation of the transverse deflecting voltage $V_{x}(\tau)$ "seen" by a trailing particle $q_{T}$ passing into the cavity after a time $\tau$ from a leading charge $q$. Assuming $q$ passing near the cavity axis, and a generic parabolic trajectory in the deflector of the type

$$
x_{q}=a_{1} z^{2}+a_{2} z+a_{3},
$$

the transverse deflecting voltage can be written as

$$
\begin{aligned}
V_{x}(\tau) & =\frac{1}{2 \pi} \int_{-\infty}^{+\infty} \tilde{V}_{x} e^{j \omega \tau} d \omega \\
& =\frac{1}{2 \pi} \int_{-\infty}^{+\infty}\left(\tilde{V}_{1} a_{1}+\tilde{V}_{2} a_{2}+\tilde{V}_{3} a_{3}\right) e^{j \omega \tau} d \omega,
\end{aligned}
$$

where

$$
\begin{aligned}
& \tilde{V}_{1}=\frac{1}{j \omega} \frac{q c}{\left(1+j Q_{L} \delta\right)} \underbrace{\frac{\int_{\text {cavity }} \frac{d E_{D z}}{d x} e^{j(\omega / c) z_{1}} d z_{1} \int_{\text {cavity }} \frac{d E_{D z}}{d x} z^{2} e^{-j(\omega / c) z} d z}{2 P_{T}}}_{R_{1}}=\frac{1}{j \omega} \frac{q c R_{1}}{\left(1+j Q_{L} \delta\right)} \\
& \tilde{V}_{2}=\frac{1}{j \omega} \frac{q c}{\left(1+j Q_{L} \delta\right)} \underbrace{\frac{\int_{\text {cavity }} \frac{d E_{D z}}{d x} e^{j(\omega / c) z_{1}} d z_{1} \int_{\text {cavity }} \frac{d E_{D z}}{d x} z e^{-j(\omega / c) z} d z}{2 P_{T}}}_{R_{2}}=\frac{1}{j \omega} \frac{q c R_{2}}{\left(1+j Q_{L} \delta\right)} \\
& \tilde{V}_{3}=\frac{1}{j \omega} \frac{q c}{\left(1+j Q_{L} \delta\right)} \underbrace{\frac{\int_{\text {cavity }} \frac{d E_{D z}}{d x} e^{j(\omega / c) z_{1}} d z_{1} \int_{\text {cavity }} \frac{d E_{D z}}{d x} e^{-j(\omega / c) z} d z}{2 P_{T}}}_{R_{3}}=\frac{1}{j \omega} \frac{q c R_{3}}{\left(1+j Q_{L} \delta\right)} .
\end{aligned}
$$

$P_{T}$ is the total dissipated power in the cavity and external load (for a given amplitude $E_{0}$ of the deflecting mode) and $Q_{L}$ is the loaded quality factor.

In the case of a pure pill-box cavity the derivative $d E_{D z} / d x$ is constant along $z$, but, in a more general case, one has to consider its longitudinal dependence to correctly calculate the previous quantities.

If we consider a symmetric cavity with respect to the plane $z=0$ it is easy to verify that $R_{1,3}$ are real while $R_{2}$ is imaginary. The corresponding induced wakefields in this case are (see Appendix A)

$$
\begin{aligned}
V_{1,3}(\tau) & \cong q c \frac{R_{1,3}}{Q_{L}} e^{-\left(\omega_{\mathrm{rf}} / 2 Q_{L}\right) \tau} \sin \left(\omega_{\mathrm{rf}} \tau\right) \\
V_{2}(\tau) & \cong q c \frac{R_{2} / j}{Q_{L}} e^{-\left(\omega_{\mathrm{rf}} / 2 Q_{L}\right) \tau} \cos \left(\omega_{\mathrm{rf}} \tau\right) .
\end{aligned}
$$

Bunches whose trajectories have zero angle at the center of the deflector $\left(a_{2}=0\right)$ generate a pure sinusoidal wakefield while particles whose trajectories have $a_{1,3}=0$ generate a cosinusoidal wakefield. Such wakefield profiles are sketched in Fig. 12. In the figure we have considered the case of two recombined trains and we have plotted the wakefield profiles generated by the first bunch of the first train assuming a general trajectory with $a_{1,2,3} \neq 0$. The trailing bunches experience both types of wakefield. The sinusoidal wakefield can give a head-tail deflecting voltage to the trailing bunches while the cosinusoidal wake can give a kick to the bunch centroids. Both the slope of the deflecting voltage and the amplitude have opposite signs for the bunches of the first and second train.

The numerical values of the coefficient $R_{1,2,3}$ can be easily calculated by e.m. codes from the geometry of the

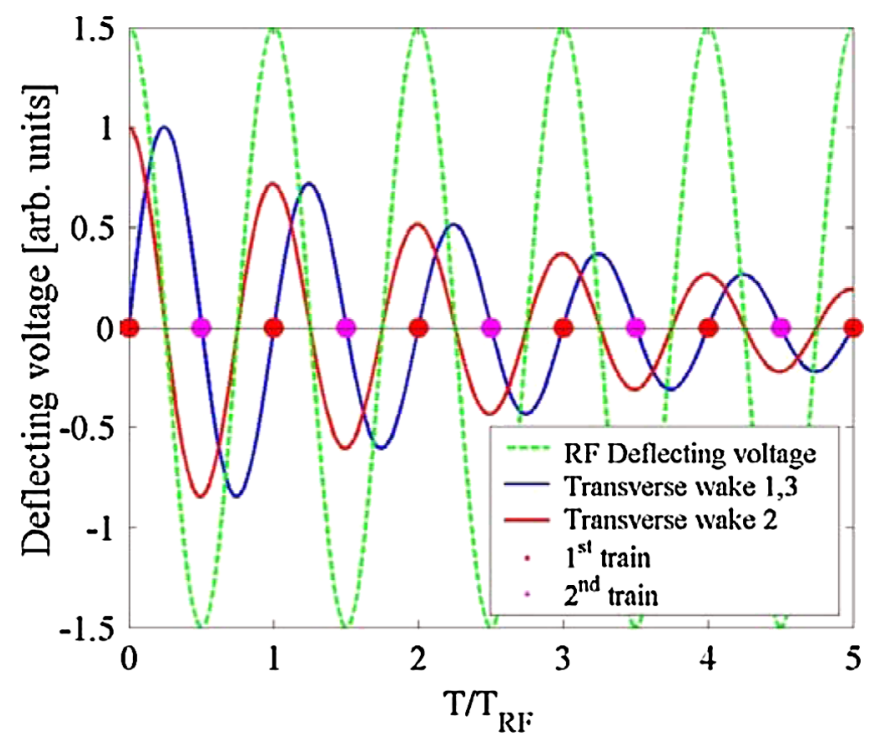

FIG. 12. (Color) Sketch of the wakefield profiles generated by the first bunch of one train assuming a general trajectory of the bunch with $a_{1,2,3} \neq 0$. In the figure we considered the case of two recombined trains of bunches (1st and 2nd train). The numbers 1, 2, and 3 are related to the different types of wakes generated by the $a_{1,2,3}$ trajectory components. 
cavity following Eqs. (4). As an example, if we consider a pure pill-box cavity the $R_{i}$ coefficients are given by (see Appendix B)

$$
\begin{aligned}
R_{1}= & \left(\frac{p_{11}}{2 a}\right)^{2} L_{c} \frac{\sin \left(\frac{L_{c}}{2} \frac{\omega_{\mathrm{rf}}}{c}\right)}{\frac{L_{c}}{2} \frac{\omega_{\mathrm{rf}}}{c}}\left[\frac{L_{c}^{2}}{2} \frac{c}{\omega_{\mathrm{rf}}} \sin \left(\frac{L_{c}}{2} \frac{\omega_{\mathrm{rf}}}{c}\right)\right. \\
& \left.+2 L_{c} \frac{c^{2}}{\omega_{\mathrm{rf}}^{2}} \cos \left(\frac{L_{c}}{2} \frac{\omega_{\mathrm{rf}}}{c}\right)-4 \frac{c^{3}}{\omega_{\mathrm{rf}}^{3}} \sin \left(\frac{L_{c}}{2} \frac{\omega_{\mathrm{rf}}}{c}\right)\right] \frac{1}{2 P_{T}} \\
R_{2}= & j\left(\frac{p_{11}}{2 a}\right)^{2} L_{c} \frac{\sin \left(\frac{L_{c}}{2} \frac{\omega_{\mathrm{rf}}}{c}\right)}{\frac{L_{c}}{2} \frac{\omega_{\mathrm{rf}}}{c}}\left[L_{c} \frac{c}{\omega_{\mathrm{rf}}} \cos \left(\frac{L_{c}}{2} \frac{\omega_{\mathrm{rf}}}{c}\right)\right. \\
& \left.-2 \frac{c^{2}}{\omega_{\mathrm{rf}}^{2}} \sin \left(\frac{L_{c}}{2} \frac{\omega_{\mathrm{rf}}}{c}\right)\right] \frac{1}{2 P_{T}} \\
R_{3}= & \left(\frac{p_{11}}{2 a}\right)^{2}\left[L_{c} \frac{\sin \left(\frac{L_{c}}{2} \frac{\omega_{\mathrm{rf}}}{c}\right)}{\frac{L_{c}}{2} \frac{\omega_{\mathrm{rf}}}{c}}\right]^{2} \frac{1}{2 P_{T}}
\end{aligned}
$$

where $L_{c}$ is the cavity length and the total dissipated power $P_{T}$ is given by

$$
\begin{aligned}
P_{T} & =\frac{\varepsilon_{0} \pi}{4 p_{11} c} J_{0}\left(p_{11}\right)^{2} \omega_{\mathrm{rf}}^{2} L_{c} a^{2}\left(1+\frac{a}{L_{c}}\right) \delta_{s}(1+\beta) \\
\delta_{s} & =\operatorname{skin} \operatorname{depth}\left(\delta_{s}=\sqrt{\frac{2}{\omega_{\mathrm{rf}} \mu_{0} \sigma}} \quad \sigma: \text { conductivity }\right) .
\end{aligned}
$$

The numerical values of such coefficients assuming a resonant frequency of $1.5 \mathrm{GHz}$ and $L_{c}=6 \mathrm{~cm}$ and those obtained in the case of the DL cavity, by direct integration of the field given by HFSS are reported in Table IV assuming $\beta=5.5$. If the bunch enters in the deflector with some initial conditions $x_{\text {in }}$ and $x_{\text {in }}{ }^{\prime}$ (at $z=-L_{c} / 2$ ) and is deflected by the transverse voltage $V_{T}$, the coefficients $a_{j}$ are simply given by

$$
\begin{gathered}
a_{1}=\frac{V_{T}}{2 E / e L_{c}} \quad a_{2}=\frac{V_{T}}{2 E / e}+x_{\mathrm{in}}^{\prime} \\
a_{3}=\frac{V_{T}}{8 E / e} L_{c}+\frac{L_{c}}{2} x_{\mathrm{in}}^{\prime}+x_{\mathrm{in}} .
\end{gathered}
$$

Their numerical values in case of $x_{\text {in }}=0$ and $x_{\text {in }}{ }^{\prime}=0$ and assuming a deflecting angle $V_{T} /(E / e)$ equal $15 \times$ $10^{-3}[\mathrm{rad}]$ are reported in Table IV. From these values it is easy to note that the dominant term in the wakefield expression is $V_{3}$.

TABLE IV. Numerical values of $R_{i}$ and $a_{i}$ coefficients.

\begin{tabular}{lrrcr}
\hline \hline & & & \multicolumn{2}{c}{$\begin{array}{c}\text { Numerical } \\
\text { value }\end{array}$} \\
\hline$R_{1}[\Omega]$ & $3.2 \times 10^{4}$ & $2.8 \times 10^{4}$ & $a_{1}\left[\mathrm{~m}^{-1}\right]$ & $12.5 \times 10^{-2}$ \\
$R_{2} / j[\Omega / \mathrm{m}]$ & $-1.2 \times 10^{6}$ & $-2.1 \times 10^{6}$ & $a_{2}$ & $7.5 \times 10^{-3}$ \\
$R_{3}\left[\Omega / \mathrm{m}^{2}\right]$ & $1.2 \times 10^{8}$ & $0.98 \times 10^{8}$ & $a_{3}[\mathrm{~m}]$ & $1.1 \times 10^{-4}$ \\
\hline \hline
\end{tabular}

\section{B. Tracking code results}

A dedicated tracking code has been written to study the multibunch multipassage effects [19].

The tracking code scheme is sketched in Fig. 13. Each bunch enters the first deflecting cavity with some horizontal initial conditions $\left(x_{\mathrm{in}}, x_{\text {in }}^{\prime}\right)$, interacts with both the main rf deflecting field and the wake left by the bunches ahead, contributes to the wake, and exits from the deflector with some new horizontal conditions $\left(x_{\text {out }_{1}}, x_{\text {out }_{1}}^{\prime}\right)$. The bunch, then, moves to the second deflecting cavity through the drift entering in it with some horizontal initial conditions $\left(x_{\mathrm{in}_{2}}, x_{\mathrm{in}_{2}}^{\prime}\right)$. It interacts with the rf field and wakes of this second device and exits from it with some new horizontal conditions $\left(x_{\text {out }_{2}}, x_{\text {out }_{2}}^{\prime}\right)$. The bunches injected into the DL are then transported again to the first deflecting cavity deflector by the transport matrix $M_{\mathrm{DL}}$. At the end of the merging process each macroparticle ends up with certain horizontal conditions ( $\left.x_{\text {out }}, x_{\text {out }}^{\prime}\right)$.

The tracking code allows therefore calculating the final values of position and angles of all bunches in the trains and its dependence on the deflector wakes, injection errors, and DL optical functions. The code can be used to study both the effects on beam dynamics due to fundamental horizontal deflecting mode and those due to the vertical one.

To take into account the effects of the finite bunch length, each bunch has been sliced into nine macroparticles

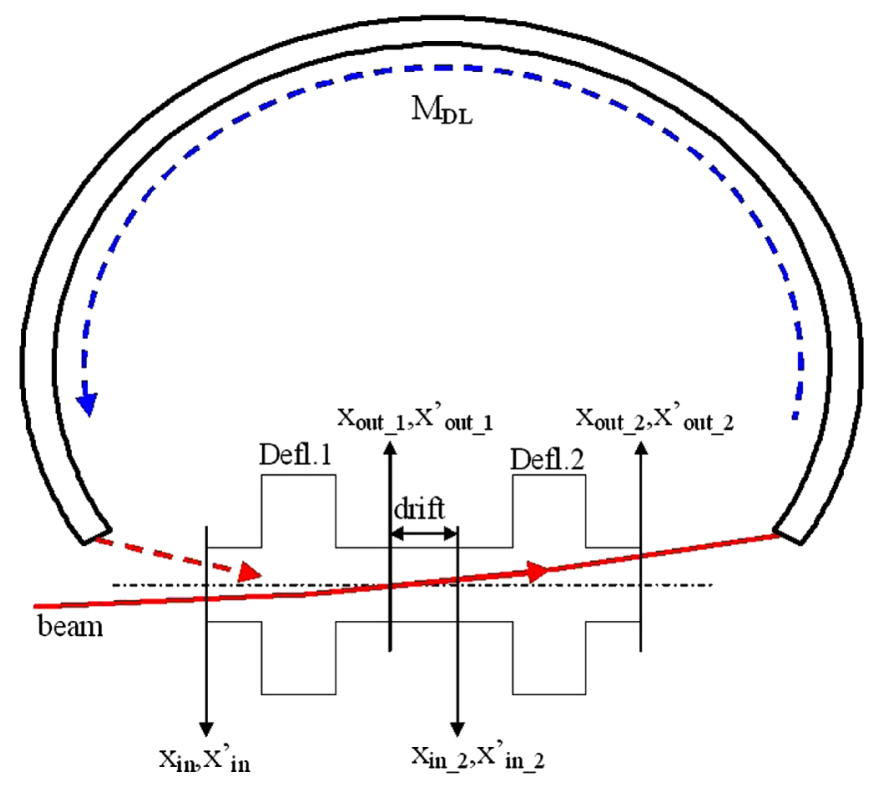

FIG. 13. (Color) Tracking code scheme. The red lines represent the particle trajectories. Each bunch enters the deflecting system with some horizontal initial conditions $\left(x_{\text {in }}, x^{\prime}{ }_{\text {in }}\right)$, interacts with both the main $\mathrm{rf}$ deflecting fields and the wakes left by the bunches ahead, contributes to the wake, and exits from the deflectors with some new horizontal conditions $\left(x_{\text {out }_{2}}, x_{\text {out }_{2}}\right)$. The bunches injected into the DL are then transported again to the first deflecting cavity deflector by the transport matrix $M_{\mathrm{DL}}$. 

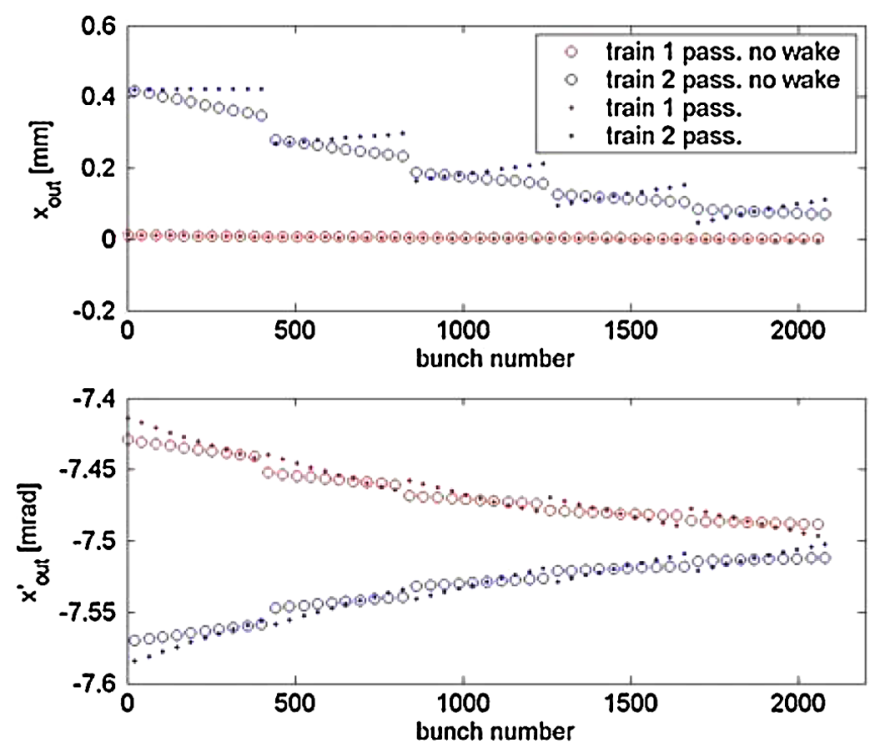

FIG. 14. (Color) Output positions and angles of bunches in the case of perfect injection.

following a Gaussian longitudinal distribution with $\sigma_{z}=$ $3 \mathrm{~mm}$. This corresponds to the maximum length of bunches passing into the cavities (see Table I) and to the worst case for the effect of the sinusoidal wakefield that results in a head-tail slope of induced deflecting voltage.

\section{Effects of the beam loading on the horizontal deflecting mode}

\section{Perfect injection of the trains}

This is the case of bunch trains injected with the initial conditions that perfectly match the main deflecting field of the deflector so that, if there were no wakes, all the bunches would end up on the design orbit. This condition corresponds to $x_{\text {in }}=0 \mathrm{~mm}$ and $x_{\text {in }}^{\prime}=7.5 \mathrm{mrad}$ and the ideal output position and angle are $x_{\text {out }}=0 \mathrm{~mm}$ and $x_{\text {out }}^{\prime}=$ $-7.5 \mathrm{mrad}$.

The output positions and angles of the central slice of the bunches with and without the effect of beam loading are shown in Fig. 14. ${ }^{3}$ The results have been obtained considering a DL phase advance $\Delta \varphi_{\mathrm{DL}}=270^{\circ}$ and an horizontal $\beta$ function at the deflectors $\beta_{D}=5 \mathrm{~m}$. Figure 15 shows the corresponding values of the Courant-Snyder invariants (CSI) of the bunch centroids, Fig. 16 the rms emittance referred to the central slice of each bunch. ${ }^{4}$ In the case of

\footnotetext{
${ }^{3}$ In the plots the "trains 2 passage" are those injected into the DL (that pass twice into the deflectors) while the "trains 1 passage" are those directly deflected into the transfer line.

${ }^{4}$ Each slice has obviously zero transverse emittance. The effect of the transverse wakefield is to spread the slices in the transverse plane. To evaluate the effect of transverse wakefield we calculate the rms projected transverse emittance following the same definition given in footnote $\mathrm{b}$ of Table I considering in the formula the differences $\left(x-x_{c}\right)$ or $\left(x^{\prime}-x_{c}^{\prime}\right)$, where $\left(x_{c}, x_{c}^{\prime}\right)$ are the transverse position and angular divergence of the central slice of each bunch.
}

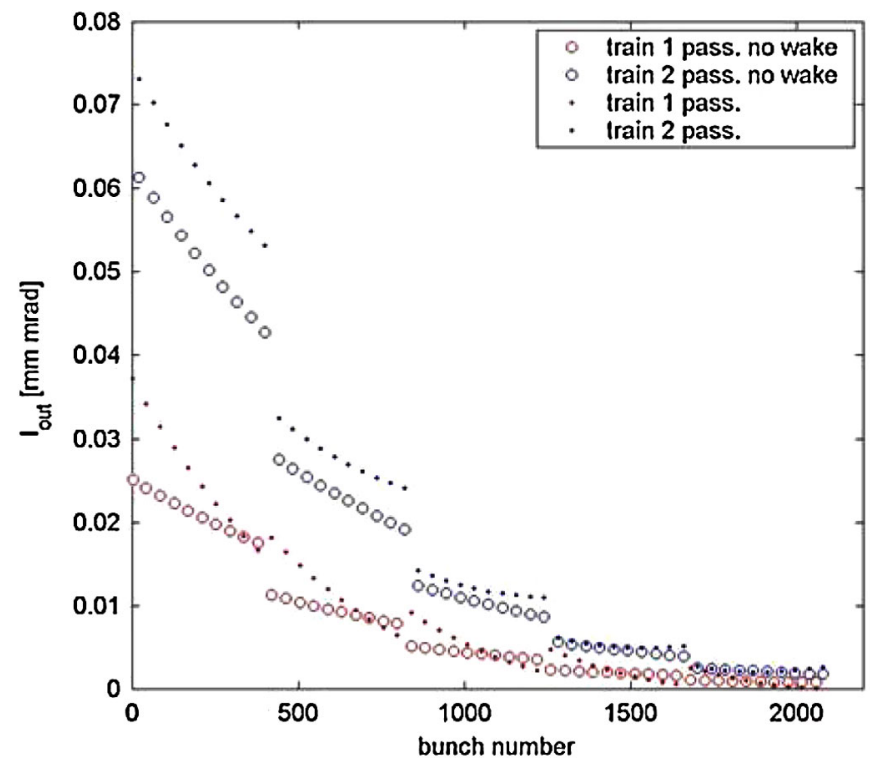

FIG. 15. (Color) Courant-Snyder invariants $\left(I_{\text {out }}\right)$ of the bunch centroids in the case of perfect injection.

no beam loading, the differences with respect to the ideal output position $x_{\text {out }}=0 \mathrm{~mm}$ and $x_{\text {out }}^{\prime}=-7.5 \mathrm{mrad}$ are due to the filling time of cavities. The plots in Figs. 14-16 show that the wakefields induced by the beam in the RFDs perturb the final output positions and angles. Nevertheless the induced spreads in position and angles are comparable with those induced by the finite filling time of cavities while the growth of the beam emittance is a small fraction $(\sim 1 \%)$ of the design one (see Table I).

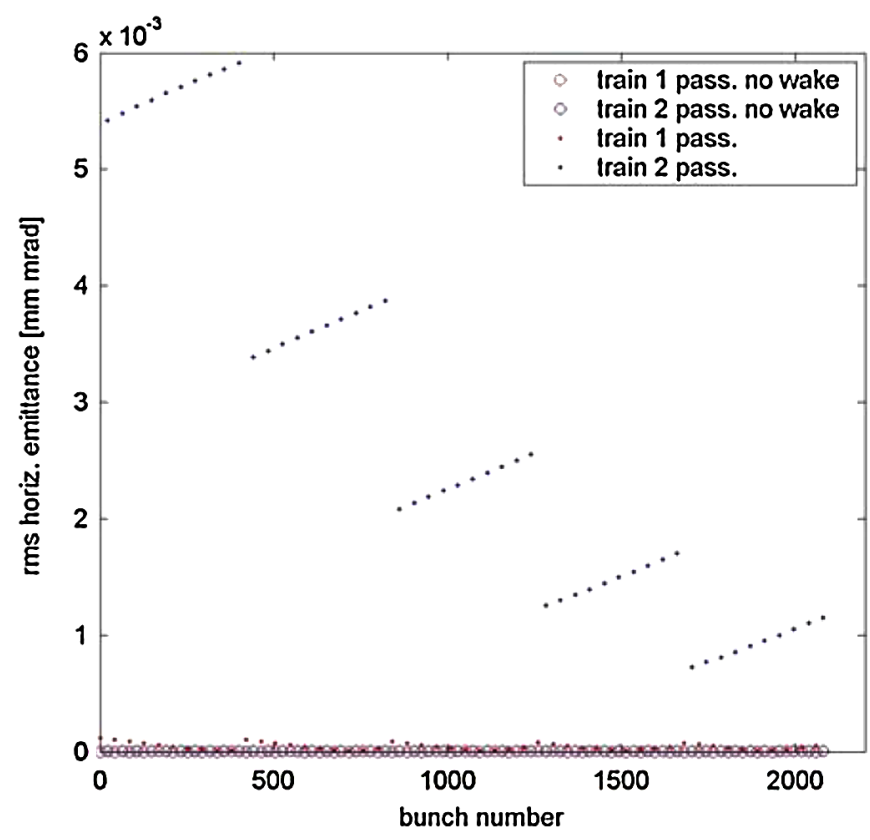

FIG. 16. (Color) The rms emittance referred to the central slice of each bunch in the case of perfect injection. 


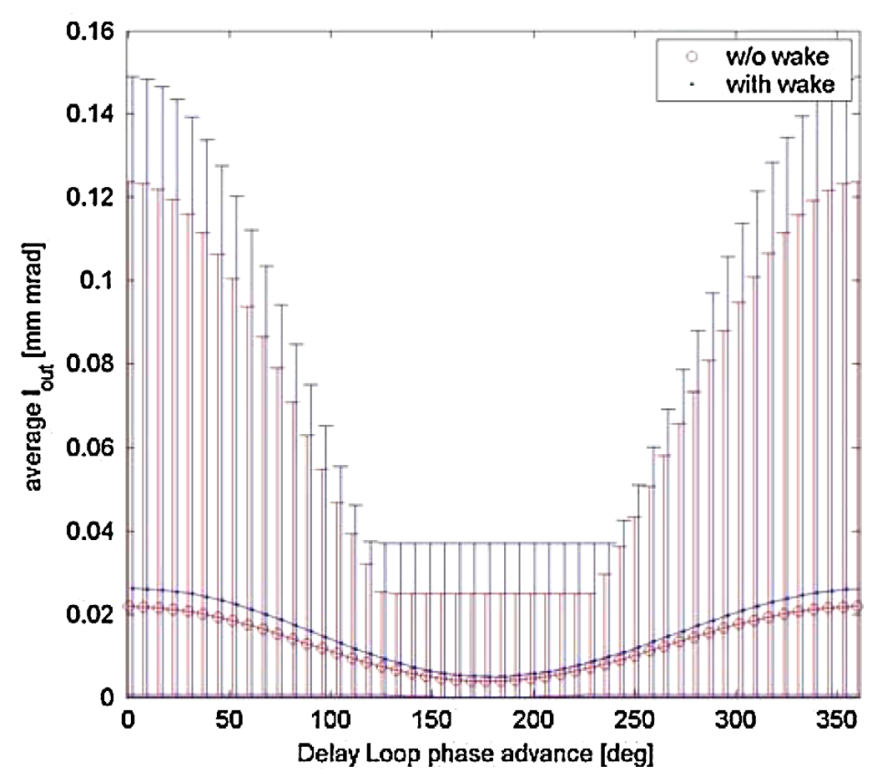

FIG. 17. (Color) Average output Courant-Snyder invariants of the bunch centroids without injection errors as a function of the DL phase advance. The bars indicate the maximum deviation from the mean value calculated over the train.

Different phase advances give different results as shown in Figs. 17 and 18, where the average CSI and rms emittances 5 are plotted as a function of the DL phase advance. In all cases, for the average positions and angles, the effects of beam loading are comparable with those of the finite filling time of cavities. In particular, if the DL phase advance is equal to $180^{\circ}$, there is a compensation effect between the first and second passage through the deflector for the "trains 2 pass" that can be intuitively understood. ${ }^{6}$ In all cases the effect of beam loading is completely negligible.

\section{Effect of injection errors}

The effect of an orbit error at injection is given in Fig. 19 where the average invariants (with respect to the case of no-wake) and the average rms emittances are shown as a function of the beam offset. We also explored the case of angular offsets at injection. All errors in injection orbit do not yield in a significant magnification of the beam loading effects. Both the emittance growth and the average invariants (with respect to the case of no-wake) are, in the worst case, a small fraction (few percent) of the CTF3 design emittance.

\footnotetext{
${ }^{5}$ In all plots we report bars that indicate the maximum and minimum deviation from the mean value calculated over the train.

${ }^{6}$ In this case, in fact, if one bunch receives a horizontal kick in the RFD lower than the nominal one (because of the finite filling time), after one turn in the delay loop, it has an angle lower than the nominal one at the entrance of the RFD. The lower deflecting voltage is, in this case, sufficient to inject the bunch on the correct orbit.
}

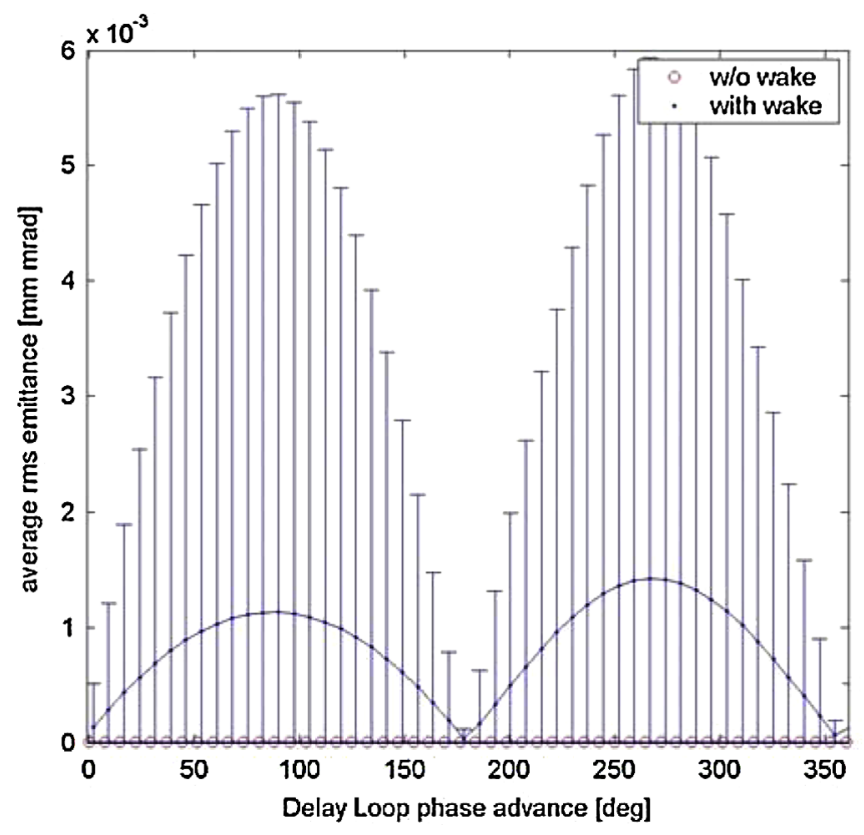

FIG. 18. (Color) The rms emittances referred to the bunch centroids without injection errors as a function of the DL phase advance. The bars indicate the maximum deviation from the mean value calculated over the train.

\section{Effects of beam loading on the vertical mode polarity}

The frequency of the deflecting mode with vertical polarity is shifted by about $40 \mathrm{MHz}$ with respect to the horizontal one because of the asymmetries in the cavity structure (coupler and beam pipe). If the beam has a vertical orbit offset inside the deflector, it can excite the vertical mode inducing vertical oscillations, while for vanishing vertical orbit offsets we do not have beam loading effects. Similar considerations as those pointed out in [20] can be done in this case. In particular, since the separation between bunches is equal to $f_{\text {rf }}$, this mode is not excited perfectly on resonance by the bunch train. This may result, for example, in a net deflecting kick on the bunch centroids of the same train due to the wakefield of type 3. Moreover, for the same reason, the filling time can become much shorter than the nominal resonant mode one $\left(2 Q / \omega_{\text {res }}\right)$.

For example, Fig. 20 shows the vertical beam offset and angles of the bunch centroids of a beam passing through the DL, and having $1 \mathrm{~mm}$ vertical offsets inside the deflector. The results have been obtained considering a DL vertical phase advance $\Delta \phi_{\mathrm{DL}}=90^{\circ}$ and a vertical $\beta$ function at the deflectors equal to $5 \mathrm{~m}$. The bunch centroids CSI calculated with respect to the case of no wake and the rms emittances referred to the central slice are reported in Figs. 21 and 22. The induced oscillation has a $\approx 40 \mathrm{MHz}$ $\Delta$ frequency with respect to the fundamental $\mathrm{rf}$ frequency of $1.5 \mathrm{GHz}$, as expected.

Figures 23 and 24 show the average centroids CSI and rms emittances with the same vertical orbit offset as a function of the DL vertical phase advance. The fact that 


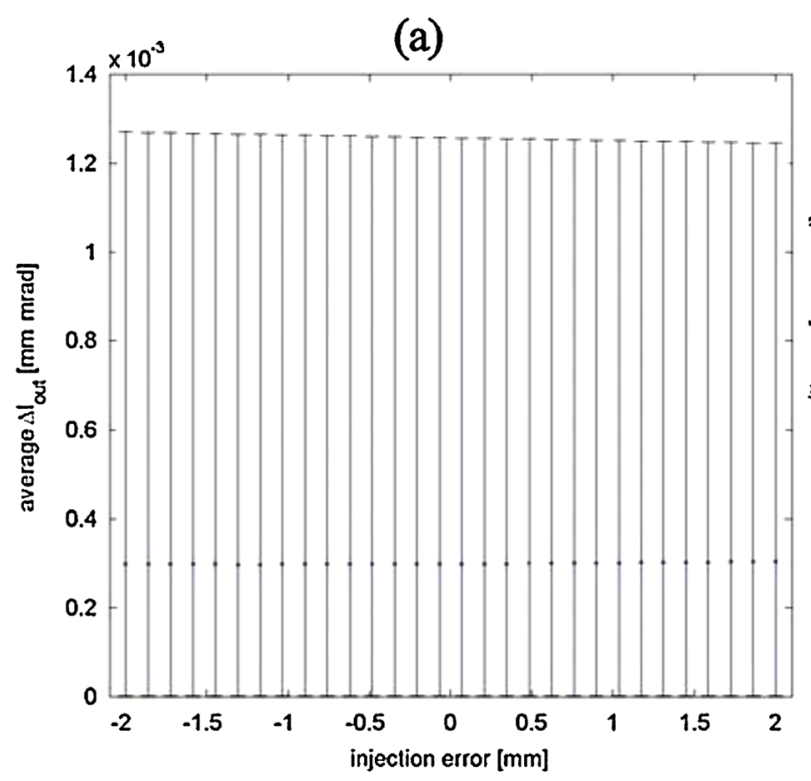

(b)

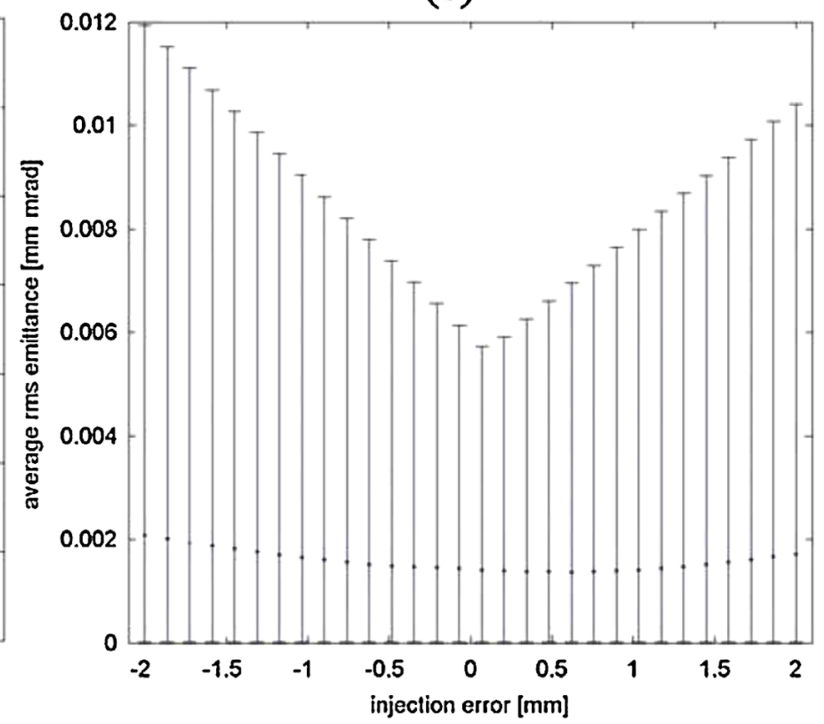

FIG. 19. (a) Average centroid invariants calculated with respect to the case of no wake in case of an orbit error at injection. The bars indicate the maximum deviation from the mean value calculated over the train. (b) Average rms emittances in case of an orbit error at injection. The bars indicate the maximum deviation from the mean value calculated over the train.

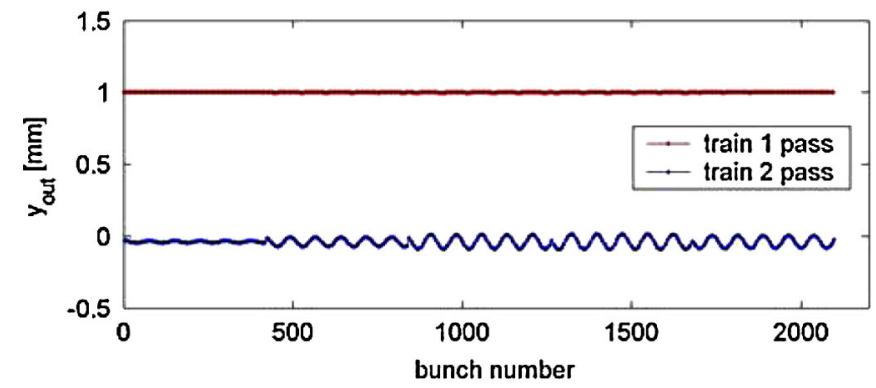

pected from the general formulas of the wakefield illustrated in Sec. III A.

Similar results can be obtained with vertical angle injection errors.

The effects on beam dynamics due to the beam loading on the vertical mode considering vertical orbit injection errors within $\pm 2 \mathrm{~mm}$ and $\pm 2 \mathrm{mrad}$ are completely tolerable. In fact both the vertical emittance growth and the

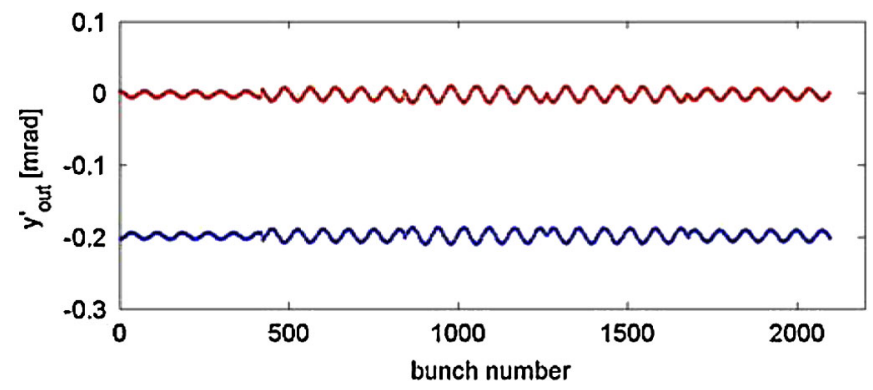

FIG. 20. (Color) Vertical beam positions and angles of the bunch centroids for a beam passing $1 \mathrm{~mm}$ vertically off axis inside the deflectors.

there is a maximum of the vertical oscillation amplitude for $\Delta \phi_{\mathrm{DL}}=180^{\circ}$ can be intuitively understood. ${ }^{7}$ Figure 25 shows the centroid CSI as a function of the vertical orbit offset. It increases quadratically with the offset, as ex-

\footnotetext{
${ }^{7}$ In this case, in fact, the vertical kicks in the two RFD passages add.
}

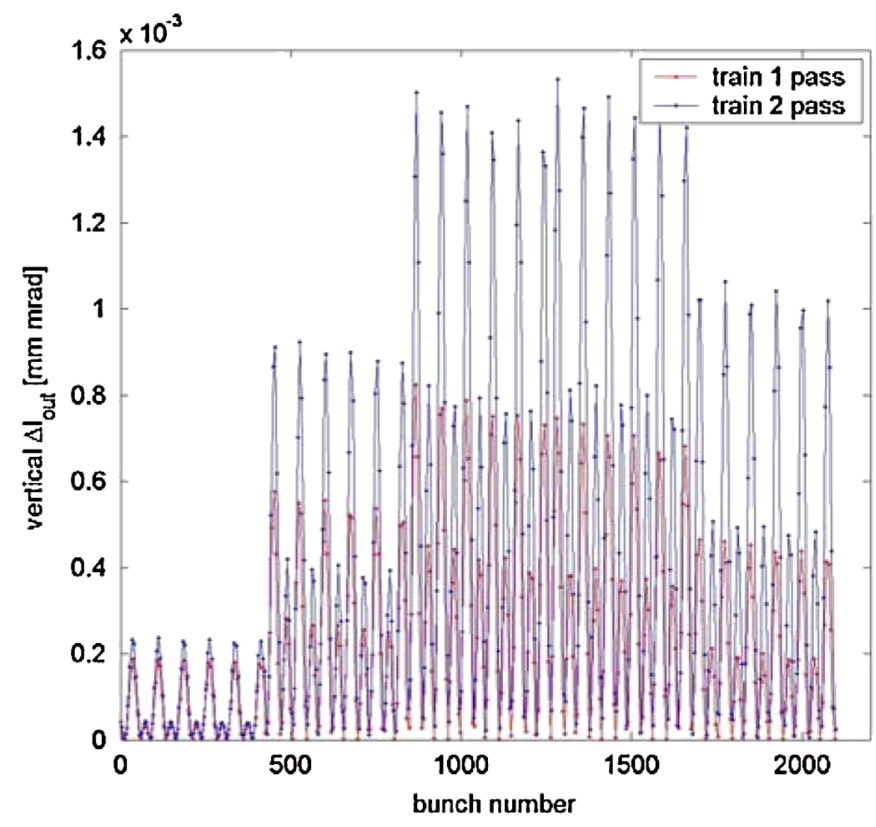

FIG. 21. (Color) Bunch centroids CSI calculated with respect to the case of no wake for a beam passing $1 \mathrm{~mm}$ off axis in the vertical plane. 


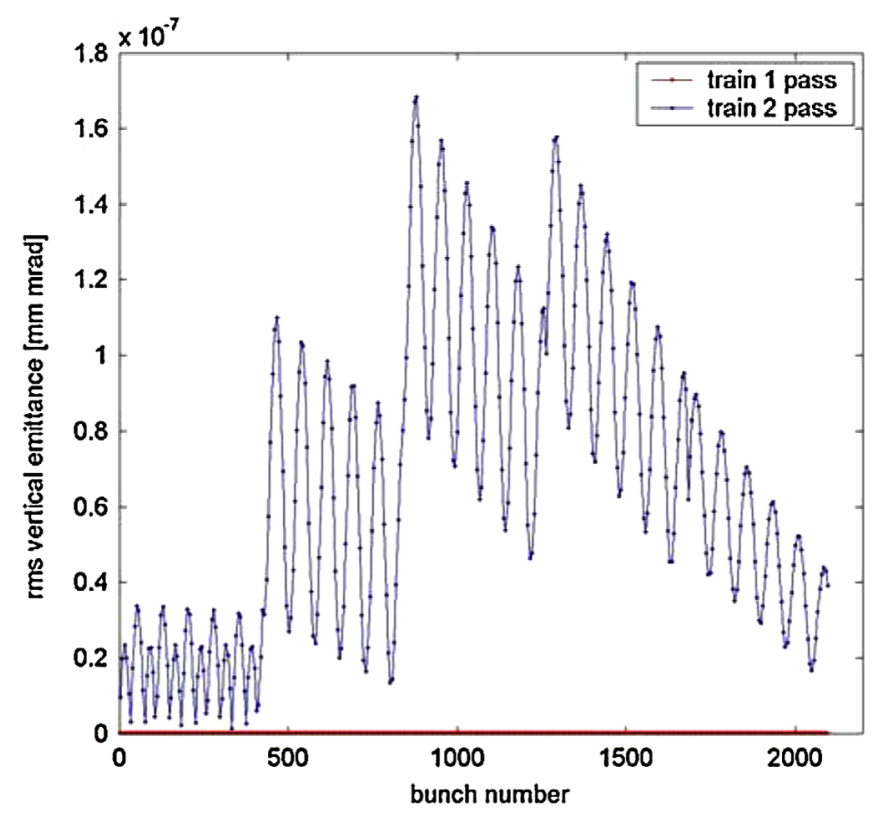

FIG. 22. (Color) Average rms emittances for a beam passing $1 \mathrm{~mm}$ off axis in the vertical plane.

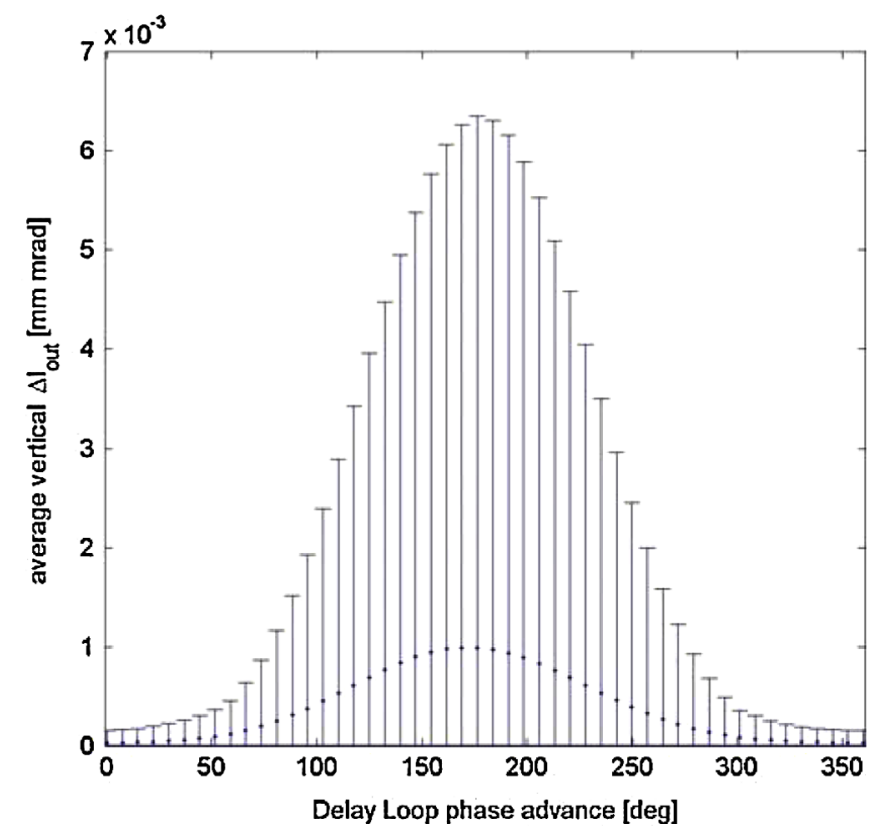

FIG. 23. Average bunch centroids CSI calculated with respect to the case of no wake for a beam passing $1 \mathrm{~mm}$ off axis in the vertical plane as a function of the DL phase advance.

average invariants (with respect to the case of no-wake) are, in the worst case, a small fraction (few percent) of the CTF3 design emittance.

\section{OPERATIONAL PERFORMANCES}

The RFD has been successfully used during the first phases of the DL commissioning and recombination has

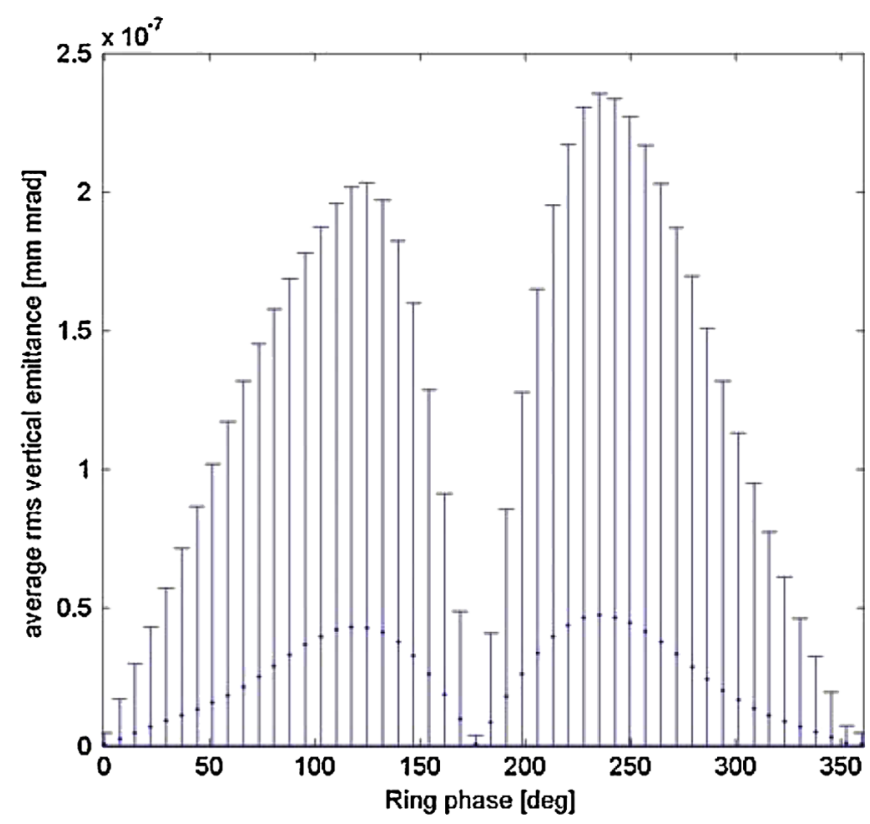

FIG. 24. Average rms vertical emittance with respect to the central slice of each bunch for a beam passing $1 \mathrm{~mm}$ off axis in the vertical plane as a function of the DL phase advance.

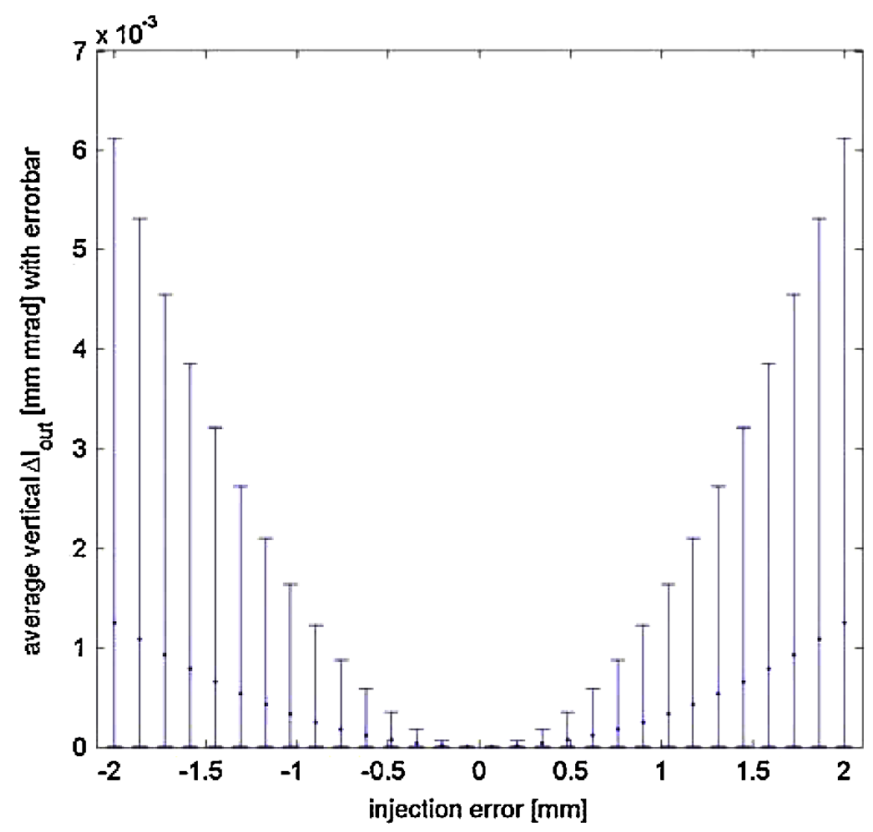

FIG. 25. Average bunch centroids CSI calculated with respect to the case of no wake as a function of the vertical orbit offset.

been successful. A detailed analysis of the DL preliminary operation with the RFD is reported in [5]. No beam instabilities due to the interaction between the bunches and the RFD have been found during operation.

The RFD can be used also as a diagnostic device to measure the bunch length: if the rf phase is $90^{\circ}$ shifted respect to normal operation condition, the bunches cross 
the cavities where the field amplitude vanishes, and time derivative is maximum. Then the field in the RFD induces a strong correlation between the particle longitudinal position in the bunch and its transverse position after the kick; a measurement of the beam profile downstream the RFD gives direct information on the bunch longitudinal length before the kick. The first results of such measurements are given in [21].

\section{CONCLUSIONS}

Three different possible solutions for the rf deflector system of the CTF3 delay loop have been considered and compared. The chosen one is a device realized with two SW resonating cavities operating on the $\mathrm{TM}_{110}$ mode connected to the rf power source through a $90^{\circ}$ hybrid junction. Such design, typically used in the SLED cavities, avoids the use of the circulator and makes the structure absolutely innovative and nonconventional. The two cavities have been overcoupled in order to have a low filling time thus reducing the spread in deflection between the head and the tail of the bunch train while the hybrid junction allows isolating the klystron from the power reflected from the cavities. The whole system has been designed using the electromagnetic code HFSS. Good results have been obtained from low power measurements and high power tests, which have confirmed the predictions of the electromagnetic simulations and the feasibility of such a new scheme.

The effects on transverse beam dynamics, both in the horizontal and the vertical planes, due to the interaction between the beam and the deflecting modes have been studied. For this purpose the single passage transverse wake induced by a charge considering different trajectories in the deflector has been calculated. A tracking code to study the multibunch multipassage effects has been implemented. The results indicate that in case of perfect injection the beam loading effects in the horizontal plane are completely negligible and are comparable with those due to the finite filling time of deflecting cavities. In case of injection errors the beam loading does not amplify significantly the initial error. Similar considerations hold in the vertical case.

The deflector system has been installed in the DL giving first successful results of train recombination and indicating that the deflector performances fulfill the expectations. According to the beam dynamics simulations no instability effects have been found during operation.

\section{ACKNOWLEDGMENTS}

All the CTF3 team at CERN gave a fundamental contribution to the realization of the deflector: several discussions with the project leader G. Geshonke and the rf group people led to the definition of parameters; special thanks to G. Mc Monagle and G. Rossat for the technical information about plants and the logistic support and S. Mathot for the suggestions concerning the brazing procedure. As in the case of the combiner ring deflector, the collaboration with the Soltan Institute for the fabrication of the cavities has given very good results. The authors would like to thank A. Gallo and L. Thorndahl for helpful discussions and suggestions and A. Ghigo, responsible for the CTF3 collaboration at LNF.

\section{APPENDIX A: TRANSVERSE WAKE FIELD IN A SW DEFLECTING CAVITY}

The general expression [22], in frequency domain, of the field excited $\left(\underline{E}_{\text {exc }}\right)$ by a current density $(J)$ flowing into the cavity on the deflection plane is

$$
\underline{E}_{\mathrm{exc}}=-\frac{1}{1+j Q_{L} \delta} \frac{\int_{\text {cavity }} J_{z} \cdot E_{D z}^{*} d V}{2 P_{T}} \underline{E}_{D},
$$

where $\delta=\left(\omega / \omega_{\text {rf }}-\omega_{\text {rf }} / \omega\right), P_{T}$ is the total dissipated power in the cavity and external load (for a given amplitude $E_{0}$ of the deflecting mode), $Q_{L}$ is the loaded quality factor, $J_{z}$ is the longitudinal component of the density current, and the symbol $*$ means the complex conjugate. ${ }^{8}$ Assuming an ultrarelativistic pointlike charge $q$ passing in $z=0$ at the time $t=0$ [see Fig. 11(b)] describing the trajectory $x=x_{q}(z)$ on the plane $(x, z)$, the corresponding current density is given by

$$
\underline{J}(t)=q \underline{s}_{0} \delta\left(t-\frac{z}{c}\right) \delta\left[x-x_{q}(z)\right] \delta(y)
$$

that in the frequency domain corresponds to

$$
\underline{J}=q \delta\left[x-x_{q}(z)\right] \delta(y) \exp (-j \omega z / c) \underline{s}_{0} .
$$

The excited field is

$$
\begin{aligned}
\underline{E}_{\mathrm{exc}} & =E_{\mathrm{excz} \underline{z}_{0}} \\
& \cong-\frac{1}{1+j Q_{L} \delta} \frac{\int_{\text {cavity }} q e^{-j(\omega / c) z} E_{D z}\left[x_{q}(z)\right] d z}{2 P_{T}} E_{D z} \underline{z}_{0} .
\end{aligned}
$$

In (A4) we have neglected the transverse component of the particle velocity assuming $\underline{s}_{0} \cong \underline{z}_{0}$.

By means of the Panofsky-Wenzel theorem [22], it is possible to calculate the transverse deflecting voltage seen by a trailing particle $q_{T}$ passing into the cavity after a time $\tau$ from the leading charge:

\footnotetext{
${ }^{8}$ For a SW cavity $E_{D z}$ is real.
} 


$$
\begin{aligned}
V_{x}(\tau) & =\frac{1}{2 \pi} \int_{\text {cavity }} \int_{-\infty}^{+\infty} \frac{\tilde{F}_{x}}{q_{T}} e^{j \omega\left(\left(z_{1} / c\right)+\tau\right)} d \omega d z_{1}=\frac{1}{2 \pi} \int_{-\infty}^{+\infty} \underbrace{\left[\int_{\text {cavity }} \frac{\tilde{F}_{x}}{q_{T}} e^{j \omega\left(z_{1} / c\right)} d z_{1}\right]}_{\tilde{V}_{x}} e^{j \omega \tau} d \omega \\
& =\frac{1}{2 \pi} \int_{-\infty}^{+\infty} \underbrace{\left[\frac{q c}{j \omega} \frac{1}{1+j Q_{L} \delta} \frac{\int_{\text {cavity }} E_{D z}\left(x_{q}\right) e^{-j(\omega / c) z} d z}{2 P_{T}} \int_{\text {cavity }} \frac{d E_{D z}}{d x} e^{j \omega\left(z_{1} / c\right)} d z_{1}\right]}_{\tilde{V}_{x}} e^{j \omega \tau} d \omega=\frac{1}{2 \pi} \int_{-\infty}^{+\infty} \tilde{V}_{x} e^{j \omega \tau} d \omega,
\end{aligned}
$$

where $\tilde{F}_{x}$ is the Fourier transform of the transverse force on the trailing charge. Equation (A5) shows that the transverse wakefield is simply given by the inverse Fourier transform of $\tilde{V}_{x}$.

Considering bunches passing near the cavity axis, it is possible to develop to first order in $x$ the $E_{D z}$ field in the form

$$
E_{D z}\left(x_{q}\right) \cong \underbrace{\frac{d E_{D z}}{d x}}_{E_{D z}^{\prime}} x_{q}
$$

Moreover, considering a generic parabolic trajectory for the leading charge in the deflector of the type expressed in Eq. (2) we obtain

$$
\tilde{V}_{x} \cong \tilde{V}_{1} a_{1}+\tilde{V}_{2} a_{2}+\tilde{V}_{3} a_{3}
$$

where the $\tilde{V}_{j}$ functions can be easily obtained giving Eqs. (4).

If we consider a symmetric cavity with respect to the plane $z=0$, the $R_{1,3}$ quantities of Eq. (4) can be approximated with the following quantities calculated at the fixed angular frequency $\omega_{\mathrm{rf}}{ }^{10}$ :

$$
\begin{aligned}
R_{1} & =\frac{\int_{\text {cavity }} E_{D z}^{\prime} e^{j(\omega / c) z_{1}} d z_{1} \int_{\text {cavity }} E_{D z}^{\prime} z^{2} e^{-j(\omega / c) z} d z}{2 P_{T}} \\
& \cong \frac{\int_{\text {cavity }} E_{D z}^{\prime} e^{j\left(\omega_{\mathrm{rf}} / c\right) z_{1}} d z_{1} \int_{\text {cavity }} E_{D z}^{\prime} z^{2} e^{-j\left(\omega_{\mathrm{rf}} / c\right) z} d z}{2 P_{T}} \\
R_{3} & =\frac{\int_{\text {cavity }} E_{D z}^{\prime} e^{j(\omega / c) z_{1}} d z_{1} \int_{\text {cavity }} E_{D z}^{\prime} e^{-j(\omega / c) z} d z}{2 P_{T}} \\
& \cong \frac{\left|\int_{\text {cavity }} E_{D z}^{\prime} e^{j\left(\omega_{\mathrm{rr}} / c\right) z} d z\right|^{2}}{2 P_{T}} .
\end{aligned}
$$

Recalling that [23]

\footnotetext{
${ }^{9} \tilde{V}_{x}$ normalized to the charge $q$ is the so-called transverse impedance.

${ }^{10}$ This approximation is usually done in the case of resonators beam coupling impedance and wakefield calculation [22].
}

$$
\begin{aligned}
& F^{-1}\left(\frac{\omega_{0}}{(\alpha+j \omega)^{2}+\omega_{0}^{2}}\right)=e^{-\alpha \tau} \sin \left(\omega_{0} \tau\right) H(\tau) \\
& F^{-1}\left(\frac{\alpha+j \omega}{(\alpha+j \omega)^{2}+\omega_{0}^{2}}\right)=e^{-\alpha \tau} \cos \left(\omega_{0} \tau\right) H(\tau),
\end{aligned}
$$

where $F^{-1}$ is the inverse Fourier transform and $H(\tau)$ is the step (or Heaviside) function, it is easy to verify that in the cases $i=1$ or $i=3$ the wakefield is simply given by

$$
\begin{aligned}
V_{1,3}(\tau) & \cong 2 q c \frac{R_{1,3}}{\sqrt{4 Q_{L}^{2}-1}} e^{-\left(\omega_{\mathrm{rf}} / 2 Q_{L}\right) \tau} \sin \left(\omega_{\mathrm{rf}} \sqrt{1-1 / 4 Q_{L}^{2}} \tau\right) \\
& \cong q c \frac{R_{1,3}}{Q_{L}} e^{-\left(\omega_{\mathrm{rf}} / 2 Q_{L}\right) \tau} \sin \left(\omega_{\mathrm{rf}} \tau\right)
\end{aligned}
$$

It is straightforward to note that the $R_{3}$ quantity is related to the transverse shunt impedance $\left(R_{T}\right)$ by the following relation:

$$
R_{3}=\left(\frac{\omega_{\mathrm{rf}}}{c}\right)^{2} R_{T}
$$

where the transverse shunt impedance $R_{T}$ is given by

$$
R_{T}=\frac{\left|\int_{0}^{L_{c}}\left(c B_{x}+E_{y}\right) e^{j \omega_{\mathrm{rf}} z / c} d z\right|^{2}}{2 P_{T}}
$$

Concerning the case $i=2$, the quantity $R_{2}$ is purely imaginary and it is possible to write

$$
\tilde{V}_{2} \cong \frac{1}{\omega_{\mathrm{rf}}} \frac{q c R_{2} / j}{\left(1+j Q_{L} \delta\right)},
$$

where $R_{2} / j$ is real and can be approximated by the following integral:

$$
R_{2} / j \cong \frac{\int_{\text {cavity }} E_{D z}^{\prime} e^{j\left(\omega_{\mathrm{rf}} / c\right) z_{1}} d z_{1} \int_{\text {cavity }} E_{D z}^{\prime} z e^{-j\left(\omega_{\mathrm{rf}} / c\right) z} d z}{j 2 P_{T}} .
$$


Recalling the expressions (A9) it is possible to write

$$
\begin{aligned}
V_{2}(\tau) \cong & q c \frac{R_{2} / j}{Q_{L}} e^{-\left(\omega_{\mathrm{rf}} / 2 Q_{L}\right) \tau} \\
& \times\left[\cos \left(\omega_{\mathrm{rf}} \sqrt{1-1 / 4 Q_{L}^{2}} \tau\right)-1 / \sqrt{4 Q_{L}^{2}-1}\right. \\
& \left.\times \sin \left(\omega_{\mathrm{rf}} \sqrt{1-1 / 4 Q_{L}^{2}} \tau\right)\right] \\
\cong & q c \frac{R_{2} / j}{Q_{L}} e^{-\left(\omega_{\mathrm{rf}} / 2 Q_{L}\right) \tau} \cos \left(\omega_{\mathrm{rf}} \tau\right) .
\end{aligned}
$$

\section{APPENDIX B: TRANSVERSE WAKEFIELD IN CASE OF A PURE PILL-BOX CAVITY}

The deflecting field components, in a pure pill-box cavity, are given by Eq. (1). Assuming $E_{0}=1$ we have

$$
\frac{d E_{D z}}{d x}=\frac{p_{11}}{2 a} .
$$

$$
\begin{aligned}
& \int_{\text {cavity }} \frac{d E_{D z}}{d x} e^{j(\omega / c) z_{1}} d z_{1}=\int_{\text {cavity }} \frac{d E_{D z}}{d x} e^{-j(\omega / c) z} d z \underbrace{\cong}_{\omega \cong \omega_{\mathrm{rf}}} \frac{p_{11}}{2 a} L_{c} \frac{\sin \left(\frac{L_{c}}{2} \frac{\omega_{\mathrm{rf}}}{c}\right)}{\frac{L_{c}}{2} \frac{\omega_{\mathrm{rf}}}{c}} \\
& \left.\int_{\text {cavity }} \frac{d E_{D z}}{d x} z e^{-j(\omega / c) z} d z \underbrace{\cong}_{\omega \cong \omega_{\mathrm{rf}}} \frac{p_{11}}{2 a}\left[\left(j \frac{z c}{\omega_{\mathrm{rf}}}+\frac{c^{2}}{\omega_{\mathrm{rf}}^{2}}\right) e^{-j\left(\omega_{\mathrm{rf}} / c\right) z}\right]\right|_{-L_{c} / 2} ^{L_{c} / 2}=j \frac{p_{11}}{2 a}\left[L_{c} \frac{c}{\omega_{\mathrm{rf}}} \cos \left(\frac{L_{c}}{2} \frac{\omega_{\mathrm{rf}}}{c}\right)-2 \frac{c^{2}}{\omega_{\mathrm{rf}}^{2}} \sin \left(\frac{L_{c}}{2} \frac{\omega_{\mathrm{rf}}}{c}\right)\right] \\
& \left.\int_{\text {cavity }} \frac{d E_{D z}}{d x} z^{2} e^{-j(\omega / c) z} d z \underbrace{\cong}_{\omega \cong \omega_{\mathrm{rf}}} \frac{p_{11}}{2 a}\left[\left(j \frac{z^{2} c}{\omega_{\mathrm{rf}}}+\frac{2 z c^{2}}{\omega_{\mathrm{rf}}^{2}}-j \frac{2 c^{3}}{\omega_{\mathrm{rf}}^{3}}\right) e^{-j\left(\omega_{\mathrm{rf}} / c\right) z}\right]\right|_{-L_{c} / 2} ^{L_{c} / 2} \\
& =\frac{p_{11}}{2 a}\left[\frac{L_{c}^{2}}{2} \frac{c}{\omega_{\mathrm{rf}}} \sin \left(\frac{L_{c}}{2} \frac{\omega_{\mathrm{rf}}}{c}\right)+2 L_{c} \frac{c^{2}}{\omega_{\mathrm{rf}}^{2}} \cos \left(\frac{L_{c}}{2} \frac{\omega_{\mathrm{rf}}}{c}\right)-4 \frac{c^{3}}{\omega_{\mathrm{rf}}^{3}} \sin \left(\frac{L_{c}}{2} \frac{\omega_{\mathrm{rf}}}{c}\right)\right] .
\end{aligned}
$$

The total dissipated power in the cavity and external load $\left(P_{T}\right)$ can be calculated as follows:

$$
P_{T}=P_{0}(1+\beta),
$$

where $\beta$ is the coupling coefficient between the cavity and the rf generator and $P_{0}$ is the power dissipated in the cavity and it is given by

$$
P_{0}=\omega_{\mathrm{rf}} \frac{W_{0}}{Q_{0}}
$$

the unloaded quality factor $Q_{0}$ is given by [12]

$$
\begin{aligned}
Q_{0} & =\frac{p_{11} c}{\omega_{\mathrm{rf}} \delta_{s}\left(1+\frac{a}{L_{c}}\right)} \\
\delta_{s} & =\operatorname{skin} \operatorname{depth}\left(\delta_{s}=\sqrt{\frac{2}{\omega_{\mathrm{rf}} \mu_{0} \sigma}} \quad \sigma: \text { conductivity }\right)
\end{aligned}
$$

while the average stored energy in the cavity $\left(W_{0}\right)$ is given by

$$
\begin{aligned}
W_{0} & =\int_{\text {cavity }} \frac{1}{2} \varepsilon_{0}|E|^{2} d V=\frac{1}{2} \varepsilon_{0} \int_{0}^{L_{c}} d z \int_{0}^{2 \pi} \cos (\vartheta)^{2} d \vartheta \int_{0}^{a} J_{1}\left(\frac{p_{11} r}{a}\right)^{2} r d r=\frac{1}{2} \varepsilon_{0} L_{c} \pi \int_{0}^{a} J_{1}\left(\frac{p_{11} r}{a}\right)^{2} r d r \\
& =\left.\frac{1}{2} \varepsilon_{0} L_{c} \pi \frac{r^{2}}{2}\left[J_{1}^{\prime}\left(\frac{p_{11} r}{a}\right)^{2}+\left(1-\frac{a^{2}}{p_{11}^{2} r^{2}}\right) J_{1}\left(\frac{p_{11} r}{a}\right)^{2}\right]\right|_{r=0} ^{r=a}=\frac{1}{2} \varepsilon_{0} L_{c} \pi \frac{a^{2}}{2} J_{1}^{\prime}\left(p_{11}\right)^{2}=\frac{1}{4} \varepsilon_{0} L_{c} \pi a^{2} J_{0}\left(p_{11}\right)^{2} .
\end{aligned}
$$

The results of Eqs. (6) and (7) can be easily found from Eqs. (B2)-(B6).

[1] CERN Report No. PS 2002-008 (RF), Geneve, 2002.

[2] J. Delahaye et al., EPAC, Stockholm, 1998.

[3] H. Braun et al., CERN Report No. 99-06, Geneve, 1999.

[4] J.P. Delaye, CLIC07 Workshop, http://clic-study.web. cern.ch/CLIC-Study/.

[5] R. Corsini et al., in Proceedings of the 10th European Particle Accelerator Conference, Edinburgh, Scotland, 2006 (EPS-AG, Edinburgh, Scotland, 2006).
[6] Z. D. Farkas et al., SLAC Report No. SLAC-PUB-1453, 1974.

[7] David Alesini and Alessandro Gallo, Phys. Rev. ST Accel. Beams 7, 034403 (2004).

[8] P. Bernard et al., CERN Report No. 68-30, 1968.

[9] A. Ghigo et al., in Proceedings of the 20th Particle Accelerator Conference, Portland, OR, 2003 (IEEE, New York, 2003), p. 2742

[10] A. Gallo et al., in Proceedings of the European Particle Accelerator Conference, Vienna, 2000 (EPS, Geneva, 2000), p. 465.

[11] F. Marcellini and D. Alesini, in Proceedings of the 9th European Particle Accelerator Conference, Lucerne, 2004 (EPS-AG, Lucerne, 2004), p. 689. 
[12] See, for example, R. E. Collin, Foundation for Microwave Engineering (McGraw-Hill, New York, 1992).

[13] http://www.ansoft.com/products/hf/hfss/.

[14] http://www.megaind.com/.

[15] http://www.thalesgroup.com/electrondevices.

[16] http://www.ipj.gov.pl/en/main.htm.

[17] F. Marcellini and D. Alesini, in Proceedings of the 10th European Particle Accelerator Conference, Edinburgh, Scotland, 2006 (Ref. [5]).

[18] See, for example, T. Wangler, RF Linear Accelerators (Wiley, New York, 1998).
[19] D. Alesini and F. Marcellini, in Proceedings of the 9th European Particle Accelerator Conference, Lucerne, 2004 (Ref. [11]), p. 2077.

[20] D. Alesini et al., in Proceedings of the 11th European Particle Accelerator Conference, Genoa, 2008 (EPS-AG, Genoa, Italy, 2008).

[21] D. Alesini et al., Report No. CTFF3-010, 2007.

[22] G. Dôme, CERN Report No. 92-03, Vol. I, 1992.

[23] See, for example, H. Dym and H. McKean, Fourier Series and Integrals (Academic Press, New York, 1985). 